\title{
Rigid Two-Step Nilpotent Lie Groups Relative to Multicontact Structures
}

\author{
IRENE VENTURI
}

ABstract - Let $\left\{X_{i}, Y_{k}\right\}$ denote a fixed basis of the Lie algebra $\mathfrak{n}$ of a connected and simply connected nilpotent Lie group $N$ of step two. Under a technical assumption on $\left\{X_{i}, Y_{k}\right\}$, we prove that the Lie algebra $\mathcal{M}$ of vector fields $V$ on $N$ that satisfy $\left[V, X_{i}\right]=\lambda_{i} X_{i}$ is finite dimensional, a property that we refer to as rigidity. Our proof allows the explicit count of $\operatorname{dim} \mathcal{M}$.

\section{Introduction.}

In a broad sense, the study of rigidity of stratified niplotent Lie groups has attracted much attention in recent years, starting from the seminal work by $\mathrm{N}$. Tanaka ([19] and references therein) and his school (notably Yamaguchi [20], Yatsui [21] and Morimoto [15]) and then moving into more explicitly geometric settings in the important papers by Korányi and Reimann [11] and Pansu [17], where various generalised contact-type structures were considered. At a more general and formal level, the notion of multicontact structure was first formulated by Cowling, De Mari, Korányi and Reimann [4,5] in the context of the "boundaries" $G / P$ of symmetric spaces $(G$ semisimple, $P$ a parabolic subgroup of $G$ ). In particular, the local nature of $G / P$ as a nilpotent stratified Lie group stemming from the Iwasawa decomposition of $G=K A N$ and from the Bruhat decomposition, together with the root structure of the Lie algebra of $N$, leads to a notion of multicontact vector field that can easily be formulated for all stratified nilpotent Lie algebras once an additional sub-stratification of the ground layer is singled out. In the case of an Iwasawa nilpotent Lie algebra $\mathfrak{n}$ in its standard form as the sum of the (restricted) positive root spaces, the stratification is provided by the height function on the root system, whereas

(*) Indirizzo dell'A.: DIMA, Università di Genova, via Dodecaneso, 35, 16146 Genova.

E-mail: venturi@dima.unige.it 
the sub-stratification refers to the simple roots. As explained by Korányi [10], the problem has a clear differential geometric counterpart: if one selects smooth distributions $\mathcal{D}_{i}$ on a smooth manifold $M$ such that the vector fields spanning the distributions satisfy a Hörmander type condition, then a multicontact mapping of $M$ is a diffeomorphism whose differential preserves each distribution $\mathcal{D}_{i}$. Passing to the infinitesimal level yields the notion of multicontact vector field, that is, a vector field $V$ on $M$ whose flow consists of multicontact mappings. If $V$ is such a vector field, the requirement amounts to the condition that $[V, X] \in \mathcal{D}_{i}$ for every $X \in \mathcal{D}_{i}$. Thus one may formulate a general problem as follows: given a stratified nilpotent Lie algebra $\mathfrak{n}=\mathfrak{n}_{1}+\cdots+\mathfrak{n}_{s}$ (parametrizing the tangent bundle of the connected and simply connected nilpotent Lie group $N$ with $\operatorname{Lie}(N)=\mathfrak{n})$ together with a multicontact structure, that is, a decomposition of $\mathfrak{n}_{1}$ into the sum of subspaces $\mathfrak{n}_{1}=\mathfrak{n}_{1}^{(1)}+\cdots+\mathfrak{n}_{1}^{(t)}$, is the Lie algebra $\mathcal{M}$ of vector fields on $N$ which preserve (under bracket) each direct summand $\mathfrak{n}_{1}^{(i)}$ finite dimensional? If yes, we say that $\mathfrak{n}$ is rigid and in this case it is natural to ask if we can describe the structure of $\mathcal{M}$. The remarkable answer in $[20]$ and $[4,5]$ is that in most cases $\mathcal{M} \simeq \operatorname{Lie}(G)$ if $M=G / P$ and if each $\mathfrak{n}_{1}^{(j)}$ is determined by the structure of $P$ in terms of simple roots. These questions are formulated by Tanaka and his school in terms of prolongation Lie algebras and rather deep results are presented in $[19,20,21]$. Other very interesting results along these lines have been obtained by Reimann on $H$-type groups [18], by Warhurst on filiform groups [22], jet spaces [23] and free algebras [24], and by Ottazzi on Hessenberg manifolds [16].

In this paper we address the following problem: if $\mathfrak{n}$ has step two and we are given certain canonical bases $\left\{X_{i}\right\}$ of $\mathfrak{n}_{1}$ and $\left\{Y_{k}\right\}$ of $\mathfrak{n}_{2}$, is $\mathfrak{n}$ rigid relative to the "finest" multicontact structure, that is, when each $\mathfrak{n}_{1}^{(i)}$ is just the span of the basis vector $X_{i}$ ? Our main theorem answers this question

positively. Its proof is based on a method suggested by Eastwood [14] and combines prolongation notions together with genuine differential geometric constructs and provides a method for bounding explicitly the dimension of $\mathcal{M}$ once the structure of $\mathfrak{n}$ is known.

\section{The multicontact system.}

2.1 - Choice of bases.

Let $\mathfrak{n}$ denote a two-step stratified nilpotent Lie algebra. This means that $\mathfrak{n}=\mathfrak{n}_{1}+\mathfrak{n}_{2}$ as a direct sum of vector spaces, where $\mathfrak{n}_{1}$ is a subspace of 
dimension $n$ which generates the $m$-dimensional subspace $\mathfrak{n}_{2}$ under bracket, that is

$$
\left[\mathfrak{n}_{1}, \mathfrak{n}_{1}\right]=\mathfrak{n}_{2} \text {. }
$$

We select bases $\left\{X_{1}, \ldots, X_{n}\right\}$ and $\left\{Y_{1}, \ldots, Y_{m}\right\}$ of $\mathfrak{n}_{1}$ and $\mathfrak{n}_{2}$, respectively, and we assume that $\left({ }^{1}\right)$

(a) $z(\mathfrak{n})=\mathfrak{n}_{2}$, that is, $z(\mathfrak{n}) \cap \mathfrak{n}_{1}=\{0\}$, where $z(\mathfrak{n})$ is the center of $\mathfrak{n}$;

(b) for every $k \in \bar{m}$ there exist $i, j \in \bar{n}$ such that $Y_{k}=\left[X_{i}, X_{j}\right]$ :

(c) for every $i \in \bar{n}$ there exist $j \in \bar{n}$ and $k \in \bar{m}$ such that $\left[X_{i}, X_{j}\right]=\gamma_{i j}^{k} Y_{k}$, where $\gamma_{i j}^{k}$ is a nonzero real constant.

Clearly, assumption (a) does not harm generality, for otherwise $\mathfrak{n}$ would split as a direct sum of an abelian Lie algebra and a nilpotent Lie algebra that does satisfy (a). Further, we can always select bases such that (b) is satisfied. Indeed, once we choose a basis $\left\{X_{1}, \ldots, X_{n}\right\}$ of $\mathfrak{n}_{1}$, the brackets $\left[X_{i}, X_{j}\right]$ generate $\mathfrak{n}_{2}$ by assumption, so we can select a basis of $\mathfrak{n}_{2}$ among them and denote its elements by $\left\{Y_{1}, \ldots, Y_{m}\right\}$. As for (c), we do not know if all two-step nilpotent Lie algebras satisfy it or not.

Observe that, by assumption (b), which is not restrictive, for any fixed $k \in \bar{m}$ there exist distinct indices $i, j \in \bar{n}$ such that $\left[X_{i}, X_{j}\right]=Y_{k}$. Hence there cannot be more directions in $\mathfrak{n}_{2}$ than distinct pairs $(i, j) \in \bar{n} \times \bar{n}$. This implies that $m \leq n(n-1) / 2$.

\subsection{1 - Examples.}

We list below two classes of two-step nilpotent Lie algebras that satisfy our hypotheses. We remark that in all these cases rigidity in our sense is essentially known (see [18] when the center has dimension at least three, and $[4,16])$. Our result can thus be seen also as a unified approach.

$H$-type algebras. For the notation and basic properties of the Heisenberg type algebras, introduced first in [6], we refer to [3]. Following standard notation, an H-type algebra is a direct sum $\mathfrak{v}+\jmath$, where $\mathfrak{v}=\mathfrak{n}_{1}$ and $z=\mathfrak{n}_{2}$, that is orthogonal with respect to a positive definite inner product $\langle\cdot, \cdot\rangle$ and for which a map $J: z \rightarrow \operatorname{End}(\mathfrak{b})$ is defined that satisfies $\left\langle J_{Y} X, X^{\prime}\right\rangle=\left\langle Y,\left[X, X^{\prime}\right]\right\rangle$ for all $Y \in z$ and all $X, X^{\prime} \in \mathfrak{v}$. Evidently, $J_{Y}$ is skew-symmetric with respect to $\langle\cdot, \cdot\rangle$. The defining condition for such an algebra to be called of Heisenberg type is that $J_{Y}^{2}=-|Y|^{2} I$ (so $J_{Y}$ is non-

$\left(^{1}\right)$ If $n$ is a positive integer, we write $\bar{n}=\{1, \ldots, n\}$. 
singular for every $Y$ ). Among the several properties of $J$, we have

$$
\left[X, J_{Y} X\right]=|X|^{2} Y
$$

for every $X \in \mathfrak{v}$ and every $Y \in \mathfrak{z}$.

Now, for a fixed $Y \neq 0$ with $|Y|=1$, the endomorphism $J_{Y}$ of $\mathfrak{v}$ is skewsymmetric and non-singular. Hence $\mathfrak{v}$ is even dimensional and we can select a basis $X_{1}, \ldots, X_{2 p}$ relative to which

$$
J_{Y}=\left[\begin{array}{cc}
0 & I_{p} \\
-I_{p} & 0
\end{array}\right] .
$$

We may obviously normalize $\left|X_{i}\right|=1$ and obtain

$$
Y=\left[X_{i}, J_{Y} X_{i}\right]= \begin{cases}-\left[X_{i}, X_{i+p}\right] & \text { if } i \leq p \\ {\left[X_{i}, X_{i-p}\right]} & \text { if } i>p,\end{cases}
$$

so that setting $Y=Y_{1}$, and completing to a basis of $z$ by choosing vectors $Y_{2}, \ldots, Y_{m}$ among the various brackets $\left[X_{i}, X_{j}\right]$, yields a basis that satisfies (b). Indeed, for every $i$ there exists either $j=i+p$ (if $i \leq p$ ) or $j=i-p$ (if $i>p)$ and $k=1$ such that $\left[X_{i}, X_{j}\right]= \pm Y_{1}$ where $\gamma_{i j}^{1}= \pm 1$.

Hessenberg algebras. This is a class of nilpotent algebras that includes algebras of arbitrary step. They were introduced first in [12], [13] and then studied in [16] from the point of view of rigidity, where it is shown that most of them are rigid, in the multicontact sense that we are interested in.

Given a simple Lie algebra $\mathfrak{g}$ with Iwasawa decomposition $\mathfrak{g}=\mathfrak{f}+\mathfrak{a}+\mathfrak{n}$ and with root system $\Sigma$ one selects a subset $\mathcal{R} \subset \Sigma^{+}$of positive roots which satisfy the following Hessenberg-type condition: if $\beta \in \mathcal{R}$ and $\alpha \in \Sigma^{+}$satisfy $\beta-\alpha \in \Sigma^{+}$, then $\beta-\alpha \in \mathcal{R}$. The most important property of Hessenberg type sets is clarified by the next proposition, which is essentially contained in all of the aforementioned references.

Proposition 2.1. A subset $\mathcal{R} \subset \Sigma^{+}$is of Hessenberg type if and only if the vector space $\mathfrak{n}_{\mathcal{R}^{\prime}}=\sum_{\gamma \in \mathcal{R}^{\prime}} \mathfrak{g}_{\gamma}$ is a (nilpotent) ideal of $\mathfrak{n}$ where $\mathcal{R}^{\prime}=\Sigma^{+} \backslash \mathcal{R}$.

Proof. Suppose $\mathcal{R} \subset \Sigma^{+}$is of Hessenberg type and let $\gamma \in \mathcal{R}^{\prime}$ and $\beta \in \mathcal{R}$. If $\beta+\gamma \in \Sigma^{+}$, then necessarily $\beta+\gamma \in \mathcal{R}^{\prime}$, for otherwise $\beta+\gamma \in \mathcal{R}$ and this implies that $\gamma \in \mathcal{R}$, contrary to the assumption. Therefore $\mathfrak{n}_{\mathcal{R}^{\prime}}$ is an ideal in $\mathfrak{n}$, obviously nilpotent.

Conversely, if $\mathfrak{n}_{\mathcal{R}^{\prime}}$ is an ideal in $\mathfrak{n}$, then it is nilpotent and the following property holds: if $\gamma \in \mathcal{R}^{\prime}$ and $\beta \in \mathcal{R}$ are such that $\beta+\gamma \in \Sigma^{+}$, then necessarily $\beta+\gamma \in \mathcal{R}^{\prime}$. This means that if $\beta+\gamma \in \mathcal{R}$ for some positive roots $\gamma$ and $\beta$, then both $\beta$ and $\gamma$ must belong to $\mathcal{R}$ for otherwise at least one of them is in $\mathcal{R}^{\prime}$ and, by assumption, so is their sum. 
We stratify $\mathfrak{n}$ by height, that is, $\mathfrak{n}_{i}$ is the sum of all root spaces of height equal to $i$, where the height refers to the number of simple roots that occur in the (unique) decomposition of a positive root as a sum of simple roots. Evidently, if $\mathcal{R}$ is of Hessenberg type, then $\mathfrak{n}_{\mathcal{R}^{\prime}}$ is a stratified ideal and therefore the quotient $\mathfrak{n}_{\mathcal{R}}=\mathfrak{n} / \mathfrak{n}_{\mathcal{R}^{\prime}}$ is again a stratified nilpotent Lie algebra, which we shall refer to as a Hessenberg algebra. Among the more interesting sets $\mathcal{R}$ of Hessenberg type are those that include all positive roots of height smaller than or equal to a positive integer $p$. If we denote by $\mathcal{R}_{p}$ such a set and let $\mathfrak{n}^{(p)}=\mathfrak{n}_{\mathcal{R}_{p}}$, the sum of the root spaces with roots in $\mathcal{R}_{p}$ then it is clear that $\mathfrak{n}^{(p)}$ has step exactly equal to $p$.

For example, if $\mathfrak{g}=\mathfrak{g l}(n, \mathbb{R})$, then $\mathcal{R}=\mathcal{R}_{2}$ may be identified with the set of indices that correspond to the first two superdiagonals of an $n \times n$ upper triangular matrix, i.e. $\{(i, j): 1 \leq j-i \leq 2\}$. Thus $\mathfrak{n}_{\mathcal{R}^{\prime}}$ consists of the upper triangular matrices whose nonzero entries can only occur in the upper-right corner whose entries $a_{i j}$ satisfy $j-i>2$, and therefore $\mathfrak{n}^{(2)}$ is the quotient of the (strictly) upper triangular $n \times n$ matrices $\mathfrak{n}$ modulo $\mathfrak{n}_{\mathcal{R}^{\prime}}$. It is natural to identify its first stratum $\mathfrak{n}_{1}^{(2)}$ with the first superdiagonal and its second stratum $\mathfrak{n}_{2}^{(2)}$ with the second superdiagonal.

Obviously, $\left\{X_{i}=E_{i, i+1}: 1 \leq i \leq n-1\right\}$ and $\left\{Y_{k}=E_{k, k+2}: 1 \leq k \leq n-2\right\}$ satisfy both (b) and (c), because

$$
\begin{aligned}
& {\left[X_{i}, X_{j}\right]= \begin{cases}Y_{i} & \text { if } j=i+1 \\
0 & \text { if } j \neq i+1\end{cases} } \\
& {\left[X_{i}, Y_{k}\right]=0 \quad \bmod \mathfrak{n}_{\mathcal{R}^{\prime}}} \\
& {\left[Y_{\ell}, Y_{k}\right]=0 \quad \bmod \mathfrak{n}_{\mathcal{R}^{\prime}} .}
\end{aligned}
$$

We remark that the dimension of $\mathfrak{n}_{1}^{(2)}$ is exactly $n-1$, which can thus be any positive integer.

\section{2 - Multicontact vector fields.}

A vector field $V$ on the connected simply connected stratified group $N$ with Lie algebra denoted by $\mathfrak{n}$ is said to be a multicontact vector field relative to the basis $\left\{X_{1}, \ldots, X_{n}\right\}$, if it satisfies

$$
\left[V, X_{i}\right]=\lambda_{i} X_{i}
$$

for all $i \in \bar{n}$ and for some smooth functions $\lambda_{1}, \ldots, \lambda_{n}$ on $N$. The vector field $V$ is not assumed to be left invariant, so we write

$$
V=\sum_{t \in \bar{n}} f_{t} X_{t}+\sum_{k \in \bar{m}} g^{k} Y_{k}
$$


where the $f_{t}$ and the $g^{k}$ are smooth functions on $N$. Here and in the following we use subscripts to indicate components along the first stratum and superscripts to indicate components along the second stratum.

In preparation for what follows we recall some basic notation used for computing Lie brackets. A bracket of elements in $\mathfrak{n}_{1}$ is of course in $\mathfrak{n}_{2}$. For $i, j \in \bar{n}$ we write

$$
\left[X_{i}, X_{j}\right]=\sum_{k \in \bar{m}} \alpha_{i j}^{k} Y_{k},
$$

where the $\alpha_{i j}^{k}$ are real constants. Evidently, $\alpha_{i j}^{k}=-\alpha_{j i}^{k}$ and $\alpha_{i j}^{k}=0$ for all $k \in \bar{m}$ if and only if $\left[X_{i}, X_{j}\right]=0$. Moreover, if $\left[X_{i}, X_{j}\right]=\gamma_{i j}^{k} Y_{k}$ as in assumption (c), we have $\alpha_{i j}^{k}=\gamma_{i j}^{k}$ and $\alpha_{i j}^{\ell}=0$ for all $\ell \neq k$. Finally, $\delta_{i j}$ stands for the Kronecker symbol.

LEMMA 2.2. The vector field $V \in \mathfrak{X}(N)$ as in (3) is a multicontact vector field on $N$ if and only if for all $i, t \in \bar{n}$ and all $k \in \bar{m}$

$$
\begin{aligned}
& X_{i}\left(g^{k}\right)=\sum_{t \in \bar{n}} \alpha_{t i}^{k} f_{t} \\
& X_{i}\left(f_{t}\right)=-\delta_{i t} \lambda_{t} .
\end{aligned}
$$

Proof. For $i \in \bar{n}$ we have

$$
\begin{aligned}
\lambda_{i} X_{i} & =\left[\sum_{t \in \bar{n}} f_{t} X_{t}+\sum_{k \in \bar{m}} g^{k} Y_{k}, X_{i}\right] \\
& =\sum_{t \in \bar{n}}\left(f_{t}\left[X_{t}, X_{i}\right]-X_{i}\left(f_{t}\right) X_{t}\right)-\sum_{k \in \bar{m}} X_{i}\left(g^{k}\right) Y_{k} \\
& =\sum_{t \in \bar{n}}\left(\sum_{k \in \bar{m}} \alpha_{t i}^{k} f_{t} Y_{k}-X_{i}\left(f_{t}\right) X_{t}\right)-\sum_{k \in \bar{m}} X_{i}\left(g^{k}\right) Y_{k} .
\end{aligned}
$$

Equating the coefficients of $X_{i}$ and $Y_{k}$ we get (5) and (6).

\section{3 - Rigidity}

In this section we prove the following

THeOREm 2.3. Under the assumption (c), the Lie algebra $\mathcal{M}$ of multicontact vector fields on a two-step stratified nilpotent connected and simply connected Lie group is finite dimensional. 


\subsection{1 - Prolongation.}

The procedure of prolongation has a long history. It may be traced back to the work of Elie Cartan and was developed by many people in several ways (see e.g. [1, 7, 8, 9, 15, 19, 20] and references therein). A formulation of prolongation in the language of exterior differential systems may be found for example in [2], while from the algebraic point of view the notion was formalized by Tanaka [19]. As explained in [1] the main idea is that " $a$ generic overdetermined system of partial differential equations may be rewritten as a first order "closed system" in which all first partial derivatives of the dependent variables are expressed in terms of the variables themselves. To do this, we must introduce extra dependent variables for unknown derivatives until all derivatives of the original and extra variables can be determined as consequences of the original equation". In this sense, a system may or may not "close up". We prove in this section that our system does indeed "close up". This can also be expressed by saying that our system admits a finite prolongation.

The linear nature of the equations that express the various "jet" variables as functions of the others allows us to prove rigidity as follows. As usual we first define a vector bundle whose fibers have as many dimensions as the number of variables that have been introduced. Then we introduce a connection on this bundle in such a way that finding a solution to the original system is equivalent to finding a covariant constant section of the bundle. Since the space of parallel sections cannot have dimension exceeding the dimension of the bundle, we infer the finite dimensionality of the space of solutions. Furthermore, equality is achieved in the flat case and we show that our connection is indeed flat, thereby obtaining precise dimension counts. Our approach is inspired by that of Eastwood [14].

In this section we focus on the heart of our proof, showing that taking sufficiently many derivatives yields a closed system. Since the computations are slightly involved, it is perhaps helpful to keep in mind the the following picture:

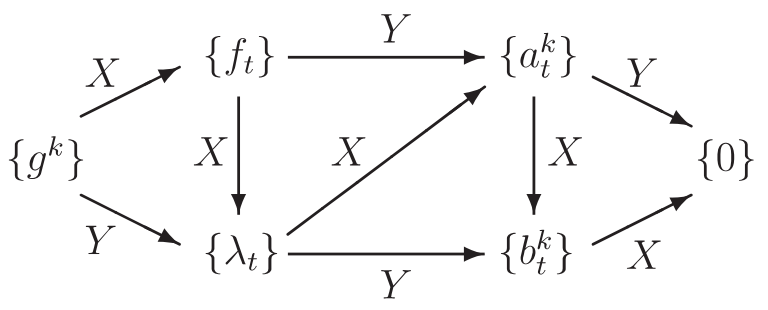


This means that we consider the real valued functions on $N$ labeled $f_{t}, g^{k}$ and $\lambda_{t}$, with $t \in \bar{n}$ and $k \in \bar{m}$. We then take derivatives with respect to $X_{i}$ and $Y_{k}$ of the functions $f_{t}, g^{k}$ and $\lambda_{t}$ and produce new variables $a_{t}^{k}$ and $b_{t}^{k}$. All $Y$-derivatives of $a_{t}^{k}$ and all $X$-derivatives of $b_{t}^{k}$ are zero for all choices of the indices if the functions $f_{t}, g^{k}$ are the coefficients of a multicontact vector field as in (3) and if the $\lambda_{t}$ satisfy (2). Hence the system associated to our multicontact problem "closes up", that is, the prolongation is finite. The extra variables are defined by

$$
\begin{array}{ll}
a_{t}^{k}=Y_{k}\left(f_{t}\right), & t \in \bar{n}, k \in \bar{m} ; \\
b_{t}^{k}=Y_{k}\left(\lambda_{t}\right), \quad & t \in \bar{n}, k \in \bar{m} .
\end{array}
$$

For computational reasons, we also introduce the variables

$$
c_{t}^{\ell k}=Y_{\ell}\left(a_{t}^{k}\right), \quad t \in \bar{n}, \ell, k \in \bar{m},
$$

although in fact they all vanish in the multicontact case (see Lemma 2.16).

From now on we shall use the symbol $\gamma_{i j}^{k}$ to mean nonzero real constants that satisfy assumption (c) without appealing explicitly to it. Evidently, we assume (5) and (6).

The following lemma describes $Y_{k}\left(g^{\ell}\right)$ for all possible pairs $(k, \ell) \in$ $\in \bar{m} \times \bar{m}$.

LEMma 2.4. For any $k, \ell \in \bar{m}$ and all $i, j \in \bar{n}$ for which $\left[X_{i}, X_{j}\right]=\gamma_{i j}^{k} Y_{k}$ we have

$$
Y_{k}\left(g^{\ell}\right)=-\delta_{k \ell}\left(\lambda_{i}+\lambda_{j}\right) .
$$

Proof. Fix $k \in \bar{m}$ and take $i, j \in \bar{n}$ such that $\left[X_{i}, X_{j}\right]=\gamma_{i j}^{k} Y_{k}$. Then for any $\ell \in \bar{m}$, by (5) and (6)

$$
\begin{aligned}
\gamma_{i j}^{k} Y_{k}\left(g^{\ell}\right) & =\left[X_{i}, X_{j}\right]\left(g^{\ell}\right) \\
& =X_{i}\left(X_{j}\left(g^{\ell}\right)\right)-X_{j}\left(X_{i}\left(g^{\ell}\right)\right) \\
& =X_{i}\left(\sum_{t \in \bar{n}} \alpha_{t j}^{\ell} f_{t}\right)-X_{j}\left(\sum_{r \in \bar{n}} \alpha_{r i}^{\ell} f_{r}\right) \\
& =-\sum_{t \in \bar{n}} \alpha_{t j}^{\ell} \delta_{i t} \lambda_{t}+\sum_{r \in \bar{n}} \alpha_{r i}^{\ell} \delta_{j r} \lambda_{r} \\
& =-\alpha_{i j}^{\ell} \lambda_{i}+\alpha_{j i}^{\ell} \lambda_{j} .
\end{aligned}
$$

Now, $\left[X_{i}, X_{j}\right]=\gamma_{i j}^{k} Y_{k}$ implies that $\alpha_{i j}^{\ell}=0$ if $\ell \neq k$, whereas if $\ell=k$, then $\alpha_{i j}^{k}=-\alpha_{j i}^{k}=\gamma_{i j}^{k} \neq 0$. 
Corollary 2.5. Let $k \in \bar{m}$ and $i, j \in \bar{n}$ be such that $\left[X_{i}, X_{j}\right]=\gamma_{i j}^{k} Y_{k}$.

(i) If there exist $p, q \in \bar{n}$ such that $\alpha_{p q}^{k} \neq 0$, then $\lambda_{i}+\lambda_{j}=\lambda_{p}+\lambda_{q}$;

(ii) if there exists $p \in \bar{n}$ with $p \neq j$ such that $\alpha_{p i}^{k} \neq 0$, then $\lambda_{j}=\lambda_{p}$.

Proof. By (4) and (7)

$$
\begin{aligned}
{\left[X_{p}, X_{q}\right]\left(g^{k}\right) } & =\sum_{\ell \in \bar{m}} \alpha_{p q}^{\ell} Y_{\ell}\left(g^{k}\right) \\
& =\alpha_{p q}^{k} Y_{k}\left(g^{k}\right)+\sum_{\ell \in \bar{m}, \ell \neq k} \alpha_{p q}^{\ell} Y_{\ell}\left(g^{k}\right) \\
& =-\alpha_{p q}^{k}\left(\lambda_{i}+\lambda_{j}\right) .
\end{aligned}
$$

But by (5) and (6)

$$
\begin{aligned}
{\left[X_{p}, X_{q}\right]\left(g^{k}\right) } & =X_{p}\left(\sum_{t \in \bar{n}} \alpha_{t q}^{k} f_{t}\right)-X_{q}\left(\sum_{r \in \bar{n}} \alpha_{r p}^{k} f_{r}\right) \\
& =-\sum_{t \in \bar{n}} \alpha_{t q}^{k} \delta_{p t} \lambda_{t}+\sum_{r \in \bar{n}} \alpha_{r p}^{k} \delta_{q r} \lambda_{r} \\
& =-\alpha_{p q}^{k}\left(\lambda_{p}+\lambda_{q}\right) .
\end{aligned}
$$

Hence, if $\alpha_{p q}^{k} \neq 0$, then $\lambda_{i}+\lambda_{j}=\lambda_{p}+\lambda_{q}$, as claimed in (i). Now, if $\alpha_{p i}^{k} \neq 0$ for some $p \neq j$, then by part (i) $\lambda_{i}+\lambda_{j}=\lambda_{i}+\lambda_{p}$, whence $\lambda_{j}=\lambda_{p}$.

Next we analyse the functions $a_{i}^{k}$, showing that the only indices $(i, k) \in \bar{n} \times \bar{m}$ for which $a_{i}^{k}$ is possibly nonzero are those for which we can find $j \in \bar{n}$ such that $\left[X_{i}, X_{j}\right]=\gamma_{i j}^{k} Y_{k}$.

Lemma 2.6. Let $i \in \bar{n}$ and $k \in \bar{m}$. If there is no $j \in \bar{n}$ such that $\left[X_{i}, X_{j}\right]=\gamma_{i j}^{k} Y_{k}$, then $a_{i}^{k}=0$.

Proof. Fix $k \in \bar{m}$. By assumption (b), there exist $\mu, v \in \bar{n}$ such that $\left[X_{\mu}, X_{v}\right]=Y_{k}$. Then for any $i \in \bar{n}$

$$
a_{i}^{k}=Y_{k}\left(f_{i}\right)=\left[X_{\mu}, X_{v}\right]\left(f_{i}\right)=X_{\mu}\left(X_{v}\left(f_{i}\right)\right)-X_{v}\left(X_{\mu}\left(f_{i}\right)\right) .
$$

Thus, if there does not exist $j \in \bar{n}$ such that $\left[X_{i}, X_{j}\right]=\gamma_{i j}^{k} Y_{k}$, then $i \notin\{\mu, v\}$ and so by (6) $X_{\mu}\left(f_{i}\right)=0=X_{v}\left(f_{i}\right)$, whence $a_{i}^{k}=0$.

Lemma 2.7. Let $i, j, t \in \bar{n}$ with $i \neq j$. Then

$$
\sum_{\ell \in \bar{m}} \alpha_{i j}^{\ell} \alpha_{t}^{\ell}= \begin{cases}0 & \text { if } t \notin\{i, j\} \\ X_{j}\left(\lambda_{i}\right) & \text { if } t=i \\ -X_{i}\left(\lambda_{j}\right) & \text { if } t=j .\end{cases}
$$


Thus, if $k \in \bar{m}$ and $i, j \in \bar{n}$ are such that $\left[X_{i}, X_{j}\right]=\gamma_{i j}^{k} Y_{k}$, then $a_{t}^{k}=0$ for $t \notin\{i, j\}, X_{i}\left(\lambda_{j}\right)=-\gamma_{i j}^{k} a_{j}^{k}$ and $X_{j}\left(\lambda_{i}\right)=\gamma_{i j}^{k} a_{i}^{k}$.

Proof. By definition of $\alpha_{t}^{\ell}$, for any $t \in \bar{n}$

$$
\sum_{\ell \in \bar{m}} \alpha_{i j}^{\ell} a_{t}^{\ell}=\sum_{\ell \in \bar{m}} \alpha_{i j}^{\ell} Y_{\ell}\left(f_{t}\right)=\left[X_{i}, X_{j}\right]\left(f_{t}\right)=X_{i}\left(X_{j}\left(f_{t}\right)\right)-X_{j}\left(X_{i}\left(f_{t}\right)\right),
$$

hence (8) follows from (6). If $\left[X_{i}, X_{j}\right]=\gamma_{i j}^{k} Y_{k}$, then the sum on the left hand side reduces to $\gamma_{i j}^{k} a_{t}^{k}$ and the conclusions follows from (6).

LEMMA 2.8. Let $i \in \bar{n}$.

(i) For any $k, \ell \in \bar{m}$ with $k \neq \ell, \sum_{t \in \bar{n}} \alpha_{t i}^{\ell} a_{t}^{k}=0$.

(ii) If $j \in \bar{n}$ and $k \in \bar{m}$ are such that $\left[X_{i}, X_{j}\right]=\gamma_{i j}^{k} Y_{k}$, then

$$
X_{i}\left(\lambda_{i}\right)=2 \gamma_{i j}^{k} a_{j}^{k}
$$

Proof. Since $\mathfrak{n}$ is two-step, for any $i \in \bar{n}$ and any $k, \ell \in \bar{m}$ we have

$$
0=\left[X_{i}, Y_{k}\right]\left(g^{\ell}\right)=X_{i}\left(Y_{k}\left(g^{\ell}\right)\right)-Y_{k}\left(X_{i}\left(g^{\ell}\right)\right) .
$$

Thus, if $\ell \neq k$, then by (7) $Y_{k}\left(g^{\ell}\right)=0$ and so using (5) and the definition of $a_{i}^{k}$

$$
0=Y_{k}\left(X_{i}\left(g^{\ell}\right)\right)=Y_{k}\left(\sum_{t \in \bar{n}} \alpha_{t i}^{\ell} f_{t}\right)=\sum_{t \in \bar{n}} \alpha_{t i}^{\ell} Y_{k}\left(f_{t}\right)=\sum_{t \in \bar{n}} \alpha_{t i}^{\ell} a_{t}^{k} .
$$

Next take $\ell=k$ and suppose that $i, j \in \bar{n}$ are such that $\left[X_{i}, X_{j}\right]=\gamma_{i j}^{k} Y_{k}$. Then by (7) we have $Y_{k}\left(g^{k}\right)=-\left(\lambda_{i}+\lambda_{j}\right)$ and so by (5) and (8) we have

$$
\begin{aligned}
0 & =\left[X_{i}, Y_{k}\right]\left(g^{k}\right) \\
& =X_{i}\left(Y_{k}\left(g^{k}\right)\right)-Y_{k}\left(X_{i}\left(g^{k}\right)\right) \\
& =-X_{i}\left(\lambda_{i}+\lambda_{j}\right)-Y_{k}\left(\sum_{t \in \bar{n}} \alpha_{t i}^{k} f_{t}\right) \\
& =-X_{i}\left(\lambda_{i}\right)+\gamma_{i j}^{k} a_{j}^{k}-\sum_{t \in \bar{n}} \alpha_{t i}^{k} a_{t}^{k} \\
& =-X_{i}\left(\lambda_{i}\right)+\gamma_{i j}^{k} a_{j}^{k}-\gamma_{j i}^{k} a_{j}^{k}-\sum_{\substack{t \in \bar{n} \\
t \neq j}} \alpha_{t i}^{k} a_{t}^{k} \\
& =-X_{i}\left(\lambda_{i}\right)+2 \gamma_{i j}^{k} a_{j}^{k}-\sum_{\substack{t \in \bar{n} \\
t \neq j}} \alpha_{t i}^{k} a_{t}^{k},
\end{aligned}
$$


where we have used that $\alpha_{j i}^{k}=-\gamma_{i j}^{k}$. Finally, by Lemma 2.7 we have $a_{t}^{k}=0$ for all $t \notin\{i, j\}$ and when $t=i$ we have $\alpha_{i i}^{k}=0$, so that all summands in the last sum vanish.

Observe that (8) and (9) describe $X_{j}\left(\lambda_{i}\right)$ for all possible pairs $(i, j) \in \bar{n} \times \bar{n}$.

Lemma 2.9. If $k \in \bar{m}$ and $i, j \in \bar{n}$ are such that $\left[X_{i}, X_{j}\right]=\gamma_{i j}^{k} Y_{k}$ and there exists $p \in \bar{n}, p \neq j$, such that $\alpha_{p i}^{k} \neq 0$, then $a_{i}^{k}=0$.

Proof. By (ii) in Corollary $2.5, \lambda_{j}=\lambda_{p}$, so that $X_{j}\left(\lambda_{j}\right)=X_{j}\left(\lambda_{p}\right)$ and thus, by Lemma 2.8 and Lemma 2.7 , respectively, we obtain

$$
-2 \gamma_{i j}^{k} a_{i}^{k}=\sum_{\ell \in \bar{m}} \alpha_{p j}^{\ell} a_{p}^{\ell}=\sum_{\substack{\ell \in \bar{m} \\ \ell \neq k}} \alpha_{p j}^{\ell} a_{p}^{\ell}
$$

since $a_{p}^{k}=0$ when $p \notin\{i, j\}$. Now, either $\alpha_{p j}^{\ell}=0$ for all $\ell \in \bar{m}$ with $\ell \neq k$, in which case $a_{i}^{k}=0$, or else there exists $\ell \in \bar{m}$ with $\ell \neq k$ such that $\alpha_{p j}^{\ell} \neq 0$. We show now that for all such indices $\ell$ we have $a_{p}^{\ell}=0$, so that the sum on the right hand side vanishes, whence the result. Fix any such $\ell$; according to Lemma 2.6 we may suppose that there exists $q \in \bar{n}$ such that $\left[X_{p}, X_{q}\right]=\gamma_{p q}^{\ell} Y_{\ell}$, for otherwise $a_{p}^{\ell}=0$. Two cases arise: either $q \neq j$ or $q=j$. In the first case, by (ii) in Corollary 2.5 necessarily $\lambda_{j}=\lambda_{q}$ and hence $\lambda_{j}=\lambda_{p}$, which gives $X_{q}\left(\lambda_{q}\right)=X_{q}\left(\lambda_{p}\right)$. By (9) and (8), respectively, we obtain

$$
2 \gamma_{q p}^{\ell} a_{p}^{\ell}=\sum_{s \in \bar{m}} \alpha_{p q}^{s} a_{p}^{s}=\gamma_{p q}^{\ell} a_{p}^{\ell}
$$

the latter equality expressing $\left[X_{p}, X_{q}\right]=\gamma_{p q}^{\ell} Y_{\ell}$. Therefore $a_{p}^{\ell}=0$ as desired. In the second case, $\left[X_{p}, X_{j}\right]=\gamma_{p j}^{\ell} Y_{\ell}$ and $\left[X_{i}, X_{j}\right]=\gamma_{i j}^{k} Y_{k}$; by Lemma 2.8, we obtain that $X_{j}\left(\lambda_{j}\right)=2 \gamma_{j p}^{\ell} a_{p}^{\ell}$ and $X_{j}\left(\lambda_{j}\right)=-2 \gamma_{i j}^{k} a_{i}^{k}$, respectively, so $2 \gamma_{j p}^{\ell} a_{p}^{\ell}=-2 \gamma_{i j}^{k} a_{i}^{k}$. Arguing as above we finally get

$$
\gamma_{p j}^{\ell} a_{p}^{\ell}=\sum_{\substack{\ell \in \bar{m} \\ \ell \neq k}} \alpha_{p j}^{\ell} a_{p}^{\ell}=-2 \gamma_{i j}^{k} a_{i}^{k}=2 \gamma_{j p}^{\ell} a_{p}^{\ell} .
$$

Therefore $a_{p}^{\ell}=0$ as desired.

LEMMA 2.10. Let $k, \ell \in \bar{m}$ with $\ell \neq k$ and let $i, j, p \in \bar{n}$ with $p \neq j$ be such that $\left[X_{i}, X_{j}\right]=\gamma_{i j}^{k} Y_{k}$ and $\left[X_{i}, X_{p}\right]=\gamma_{i p}^{\ell} Y_{\ell}$, then $a_{i}^{s}=0$ for any $s \in \bar{m}$. 
Proof. By Lemma 2.7 and item (i) of Lemma 2.8

$$
\begin{aligned}
& 0=\sum_{s \in \bar{m}} \alpha_{s j}^{k} a_{s}^{\ell}=\gamma_{i j}^{k} a_{i}^{\ell}+\alpha_{p j}^{k} a_{p}^{\ell} \\
& 0=\sum_{s \in \bar{m}} \alpha_{s p}^{\ell} a_{s}^{k}=\gamma_{i p}^{\ell} a_{i}^{k}+\alpha_{j p}^{\ell} a_{j}^{k} .
\end{aligned}
$$

We show now that $a_{i}^{\ell}=0$ whenever $\ell \neq k$. This is obvious from the first equation if $\alpha_{p j}^{k}=0$. Suppose then $\alpha_{p j}^{k} \neq 0$. By item (ii) of Corollary 2.5, necessarily $\lambda_{i}=\lambda_{p}$, which gives $X_{i}\left(\lambda_{i}\right)=X_{i}\left(\lambda_{p}\right)$. By (9) and (8), respectively, we obtain

$$
2 \gamma_{i p}^{\ell} a_{p}^{\ell}=\gamma_{p i}^{\ell} a_{p}^{\ell} .
$$

Therefore $a_{p}^{\ell}=0$ and from the first equation $a_{i}^{\ell}=0$, as desired.

Next we show that $a_{i}^{k}=0$. This is obvious from the second equation if $\alpha_{j p}^{\ell}=0$. Suppose then $\alpha_{j p}^{\ell} \neq 0$. By item (ii) of Corollary 2.5, necessarily $\lambda_{i}=\lambda_{j}$, which gives $X_{i}\left(\lambda_{i}\right)=X_{i}\left(\lambda_{j}\right)$. By (9) and (8), respectively, we obtain

$$
2 \gamma_{i j}^{k} a_{j}^{k}=-\gamma_{i j}^{k} a_{j}^{k} .
$$

Therefore $a_{j}^{k}=0$ and from the second equation $a_{i}^{k}=0$, as desired.

Finally, take $s \in \bar{m}$ for every $s \notin\{\ell, k\}$. If there exists no $q \in \bar{n}$ such that $\left[X_{i}, X_{q}\right]=\gamma_{i q}^{s} Y_{s}$, then $a_{i}^{s}=0$ by Lemma 2.6. Conversely, if $\left[X_{i}, X_{q}\right]=$ $=\gamma_{i q}^{s} Y_{s}$ for some $q \in \bar{n}$ then the previous argument with $q$ in place of $p$ and $s$ in place of $\ell$ gives $a_{i}^{s}=0$.

Observe that by Lemma 2.6, Lemma 2.7 and Lemma 2.10 the structure of the $m \times n$ matrix $a=\left(a_{i}^{k}\right)$ is subject to many constraints. Indeed, if we fix both the row index $k$ and the column index $i$ and we cannot find another column index $j$ for which $\left[X_{i}, X_{j}\right]=\gamma_{i j}^{k} Y_{k}$, then Lemma 2.6 says that $a_{i}^{k}=0$. Therefore for any fixed row $k$, the only column indices $i$ for which $a_{i}^{k}$ is possibly nonzero are those for which $\left[X_{i}, X_{j}\right]=\gamma_{i j}^{k} Y_{k}$ for some $j$. Take such a pair $(i, j)$. By Lemma 2.7 any other $a_{t}^{k}$ vanishes. Hence for any fixed row $k$ there is at most a pair of column indices $i$ and $j$ for which the corresponding entries do not vanish, i.e., every row contains at most two nonzero entries. But more is true: if for two different rows, say the $k^{\text {th }}$ and the $\ell^{\text {th }}$, we find a coinciding column index, say $i$, then by Lemma 2.10 we have $a_{i}^{s}=0$ for every $s$. Therefore, every column contains at most one nonzero entry. It follows that, after reordering, we may assume that the matrix $a=\left(a_{i}^{k}\right)$ has the following form 


$$
\left(\begin{array}{ccccccc|ccc|ccc}
* & * & 0 & 0 & \cdots & 0 & 0 & 0 & \cdots & 0 & 0 & \cdots & 0 \\
0 & 0 & * & * & \cdots & 0 & 0 & \vdots & & \vdots & \vdots & & \vdots \\
\vdots & \vdots & \vdots & \vdots & & \vdots & \vdots & \vdots & & \vdots & \vdots & & \vdots \\
0 & 0 & 0 & 0 & \cdots & * & * & 0 & \cdots & 0 & 0 & \cdots & 0 \\
\hline 0 & \cdots & \cdots & \cdots & \cdots & \cdots & 0 & * & \cdots & 0 & 0 & \cdots & 0 \\
\vdots & & & & & & \vdots & \vdots & & \vdots & \vdots & & \vdots \\
0 & \cdots & \cdots & \cdots & \cdots & \cdots & 0 & 0 & \cdots & * & 0 & \cdots & 0 \\
\hline 0 & \cdots & \cdots & \cdots & \cdots & \cdots & 0 & 0 & \cdots & 0 & 0 & \cdots & 0 \\
\vdots & & & & & & \vdots & \vdots & & \vdots & \vdots & & \vdots \\
0 & \cdots & \cdots & \cdots & \cdots & \cdots & 0 & 0 & \cdots & 0 & 0 & \cdots & 0
\end{array}\right) .
$$

The stars refer to nonzero entries; evidently, each block of rows (those with two, those with one and those with no nonzero entries) could be empty. Next we investigate the functions $b_{i}^{k}$.

Lemma 2.11. Let $i, t \in \bar{n}$ and $k \in \bar{m}$. Then

$$
X_{i}\left(a_{t}^{k}\right)=-\delta_{i t} b_{t}^{k}
$$

and if there is no $j \in \bar{n}$ such that $\left[X_{i}, X_{j}\right]=\gamma_{i j}^{k} Y_{k}$, then $X_{i}\left(a_{i}^{k}\right)=-b_{i}^{k}=0$.

Proof. Since $\mathfrak{n}$ is step-two, using the definitions of $a_{i}^{k}, b_{i}^{k}$, and (6)

$$
\begin{aligned}
0 & =\left[X_{i}, Y_{k}\right]\left(f_{t}\right) \\
& =X_{i}\left(Y_{k}\left(f_{t}\right)\right)-Y_{k}\left(X_{i}\left(f_{t}\right)\right) \\
& =X_{i}\left(a_{t}^{k}\right)+\delta_{i t} Y_{k}\left(\lambda_{t}\right) \\
& =X_{i}\left(a_{t}^{k}\right)+\delta_{i t} b_{t}^{k},
\end{aligned}
$$

thereby showing (10). It follows from Lemma 2.6 that if there is no $j \in \bar{n}$ such that $\left[X_{i}, X_{j}\right]=\gamma_{i j}^{k} Y_{k}$, then $a_{i}^{k}=0$, in which case (10) implies $0=X_{i}\left(a_{i}^{k}\right)=-b_{i}^{k}$.

Observe that (10) describes $X_{i}\left(a_{j}^{k}\right)$ for all possible triples $(i, j, k) \in$ $\in \bar{n} \times \bar{n} \times \bar{m}$. Also, Lemma 2.6 says that if there is no $j \in \bar{n}$ such that $\left[X_{i}, X_{j}\right]=\gamma_{i j}^{k} Y_{k}$, then $a_{i}^{k}=0$; under these circumstances, because of (10), we have a fortiori that $b_{i}^{k}=0$. 
Lemma 2.12. If $k \in \bar{m}$ and $i, j \in \bar{n}$ are such that $\left[X_{i}, X_{j}\right]=\gamma_{i j}^{k} Y_{k}$ then $b_{i}^{k}=b_{j}^{k}$.

Proof. Since $\left[X_{i}, X_{j}\right]=\gamma_{i j}^{k} Y_{k}$, (8) gives

$$
X_{j}\left(\lambda_{i}\right)=\sum_{\ell \in \bar{m}} \alpha_{i j}^{\ell} a_{i}^{\ell}=\gamma_{i j}^{k} a_{i}^{k} .
$$

By the definitions of $b_{i}^{k}, b_{j}^{k}$ and by (9) and (10)

$$
\begin{aligned}
\gamma_{i j}^{k} b_{i}^{k} & =Y_{k}\left(\lambda_{i}\right) \\
& =\left[X_{i}, X_{j}\right]\left(\lambda_{i}\right) \\
& \left.=X_{i}\left(X_{j}\left(\lambda_{i}\right)\right)\right)-X_{j}\left(X_{i}\left(\lambda_{i}\right)\right) \\
& =X_{i}\left(\gamma_{i j}^{k} a_{i}^{k}\right)-X_{j}\left(2 \gamma_{i j}^{k} a_{j}^{k}\right) \\
& =-\gamma_{i j}^{k} b_{i}^{k}+2 \gamma_{i j}^{k} b_{j}^{k},
\end{aligned}
$$

where $\gamma_{i j}^{k} \neq 0$, hence the result.

Lemma 2.13. Let $i, j, t \in \bar{n}$ with $i \neq j$ and $t \notin\{i, j\}$. Then

$$
\sum_{\ell \in \bar{m}} \alpha_{i j}^{\ell} b_{t}^{\ell}=0 \text {. }
$$

Thus, if $k \in \bar{m}$ and $i, j \in \bar{n}$ are such that $\left[X_{i}, X_{j}\right]=\gamma_{i j}^{k} Y_{k}$, then $b_{t}^{k}=0$ for $t \notin\{i, j\}$.

Proof. Apply $X_{t}$ to the left hand side of (8), use (10) and Lemma 2.7.

Lemma 2.14. Let $k \in \bar{m}$ and $i, j \in \bar{n}$ be such that $\left[X_{i}, X_{j}\right]=\gamma_{i j}^{k} Y_{k}$. If there exists $p \in \bar{n}, p \neq j$, such that $\alpha_{p i}^{k} \neq 0$, then $b_{i}^{k}=0$.

Proof. By Lemma $2.9 a_{i}^{k}=0$. Applying $X_{i}$ and (10) to $a_{i}^{k}=0$ we obtain that $b_{i}^{k}=0$.

LEMMA 2.15. Let $k, \ell \in \bar{m}$ with $\ell \neq k$ and $i, j, p \in \bar{n}$ with $p \neq j$ be such that $\left[X_{i}, X_{j}\right]=\gamma_{i j}^{k} Y_{k}$ and $\left[X_{i}, X_{p}\right]=\gamma_{i p}^{\ell} Y_{\ell}$ then

(i) $b_{i}^{s}=0$ for any $s \in \bar{m}$;

(ii) $b_{q}^{k}=b_{q}^{\ell}=0$ for any $q \in \bar{n}$. 
Proof. By Lemma $2.10 a_{i}^{s}=0$. Applying $X_{i}$ and (10) to $a_{i}^{s}=0$ we obtain that $b_{i}^{s}=0$ for any $s \in \bar{m}$. This proves (i). As for (ii), first of all we know from Lemma 2.13 that $b_{q}^{k}=0$ if $q \notin\{i, j\}$ and $b_{q}^{\ell}=0$ if $q \notin\{i, p\}$. As for the remaining indices,by part (i) $b_{i}^{s}=0$ for any $s \in \bar{m}$ and by Lemma 2.12 $b_{j}^{k}=b_{i}^{k}=0$ and $b_{p}^{\ell}=b_{i}^{\ell}=0$.

Lemma 2.11 tells us that the matrix $b=\left(b_{i}^{k}\right)$ has the same general shape as the matrix $a=\left(a_{i}^{k}\right)$. Furthermore, Lemma 2.12 says that whenever a row contains two nonzero entries, they actually coincide. Finally, Lemma 2.15 implies that no row may contain a single nonzero entry. The general shape of the $b$ matrix is thus

$$
\left(\begin{array}{ccccccc|ccc}
* & * & 0 & 0 & \cdots & 0 & 0 & 0 & \cdots & 0 \\
0 & 0 & * & * & \cdots & 0 & 0 & \vdots & & \vdots \\
\vdots & \vdots & \vdots & \vdots & & \vdots & \vdots & \vdots & & \vdots \\
0 & 0 & 0 & 0 & \cdots & * & * & 0 & \cdots & 0 \\
\hline 0 & \cdots & \cdots & \cdots & \cdots & \cdots & 0 & 0 & \cdots & 0 \\
\vdots & & & & & & \vdots & \vdots & & \vdots \\
0 & \cdots & \cdots & \cdots & \cdots & \cdots & 0 & 0 & \cdots & 0
\end{array}\right) .
$$

Lemma 2.16. For all $t \in \bar{n}$ and all $\ell, k \in \bar{m} c_{t}^{\ell k}=0$. For all $i, j \in \bar{n}$ and all $k \in \bar{m}$

$$
X_{j}\left(b_{i}^{k}\right)=0 .
$$

Proof. Assume first $i \neq j$. By Lemma 2.11 we have $X_{i}\left(a_{i}^{k}\right)=-b_{i}^{k}$ and $X_{j}\left(a_{i}^{k}\right)=0$. Hence,

$$
X_{j}\left(b_{i}^{k}\right)=X_{i}\left(X_{j}\left(a_{i}^{k}\right)\right)-X_{j}\left(X_{i}\left(a_{i}^{k}\right)\right)=\left[X_{i}, X_{j}\right]\left(a_{i}^{k}\right)=\sum_{\ell \in \bar{m}} \alpha_{i j}^{\ell} Y_{\ell}\left(a_{i}^{k}\right)=\sum_{\ell \in \bar{m}} \alpha_{i j}^{\ell} c_{i}^{\ell k} .
$$

In particular, if $k \in \bar{m}$ and $i, j \in \bar{n}$ are such that $\left[X_{i}, X_{j}\right]=\gamma_{i j}^{k} Y_{k}$, then $X_{j}\left(b_{i}^{k}\right)=\gamma_{i j}^{k} c_{i}^{k k}$ and by Lemma 2.12 they are both equal to $X_{j}\left(b_{j}^{k}\right)$. Similarly, $X_{i}\left(b_{i}^{k}\right)=X_{i}\left(b_{j}^{k}\right)=-\gamma_{i j}^{k} c_{j}^{k}$. Hence (11) will follow (for all possible indices $i, j$ and $k$ ) from the fact that $c_{t}^{\ell k}=0$ for all $t \in \bar{n}$ and all $\ell, k \in \bar{m}$.

Fix now $k, \ell \in \bar{m}$. By assumption (b) there exist $i, j \in \bar{n}$ such that $\left[X_{i}, X_{j}\right]=\gamma_{i j}^{k} Y_{k}$. We show next that for any $\ell \in \bar{m}$ we have $c_{t}^{\ell k}=c_{t}^{k \ell}=0$ whenever $t \notin\{i, j\}$. Indeed, by Lemma $2.7 a_{t}^{k}=0$ for $t \notin\{i, j\}$, so that using the definition we obtain $c_{t}^{\ell k}=Y_{\ell}\left(a_{t}^{k}\right)=0$ for any $\ell \in \bar{m}$. Further, by 
definition and by (10)

$$
\gamma_{i j}^{k} c_{t}^{k \ell}=\gamma_{i j}^{k} Y_{k}\left(a_{t}^{\ell}\right)=X_{i}\left(X_{j}\left(a_{t}^{\ell}\right)\right)-X_{j}\left(X_{i}\left(a_{t}^{\ell}\right)\right)= \begin{cases}0 & t \notin\{i, j\} \\ X_{j}\left(b_{i}^{\ell}\right) & t=i \\ -X_{i}\left(b_{j}^{\ell}\right) & t=j,\end{cases}
$$

whose first line proves our claim.

Hereafter we fix $k \in \bar{m}$ and we assume that $i, j \in \bar{n}$ are such that $\left[X_{i}, X_{j}\right]=\gamma_{i j}^{k} Y_{k}$. We show next that $c_{i}^{k k}=c_{j}^{k k}=0$. By definition of $b_{i}^{k}, c_{j}^{k k}$, by (9) and Lemma 2.12

$$
\begin{aligned}
0 & =\left[X_{i}, Y_{k}\right]\left(\lambda_{i}\right) \\
& =X_{i}\left(b_{i}^{k}\right)-Y_{k}\left(2 \gamma_{i j}^{k} a_{j}^{k}\right) \\
& =X_{i}\left(b_{j}^{k}\right)-2 Y_{k}\left(\gamma_{i j}^{k} a_{j}^{k}\right) \\
& =-\gamma_{i j}^{k} c_{j}^{k k}-2 \gamma_{i j}^{k} c_{j}^{k k},
\end{aligned}
$$

the latter equality following from $X_{i}\left(b_{j}^{k}\right)=-\gamma_{i j}^{k} c_{j}^{k k}$, which was shown above. This proves that $c_{j}^{k k}=0$ and an analogous argument shows that $c_{i}^{k k}=0$.

Finally we show that whenever $\ell \neq k$ we have $c_{t}^{\ell k}=c_{t}^{k \ell}=0$ for $t \in\{i, j\}$ hence proving the lemma. To this end, we observe that by assumption (b) we may choose $p, q \in \bar{n}$ such that $\left[X_{p}, X_{q}\right]=\gamma_{p q}^{\ell} Y_{\ell}$ and two cases arise: either $\{p, q\} \cap\{i, j\}=\emptyset$ or, by symmetry we may assume $p=i$. In the first case, Lemma 2.13 implies $b_{i}^{\ell}=b_{j}^{\ell}=0$ and hence computing as above further implies that

$$
\gamma_{i j}^{k} c_{t}^{k \ell}=\gamma_{i j}^{k} Y_{k}\left(a_{t}^{\ell}\right)= \begin{cases}0 & t \notin\{i, j\} \\ X_{j}\left(b_{i}^{\ell}\right) & t=i \\ -X_{i}\left(b_{j}^{\ell}\right) & t=j\end{cases}
$$

we get that the second and third lines vanish, whence $c_{t}^{k \ell}=0$ for all $t \in \bar{n}$. Similarly, by Lemma $2.13, b_{p}^{k}=b_{q}^{k}=0$ and hence computing as above that $c_{t}^{\ell k}=0$ for all $t \in \bar{n}$. In the second case, that is $p=i$, we compute that

$$
\gamma_{i j}^{k} c_{t}^{k \ell}=\gamma_{i j}^{k} Y_{k}\left(a_{t}^{\ell}\right)=X_{i}\left(X_{j}\left(a_{t}^{\ell}\right)\right)-X_{j}\left(X_{i}\left(a_{t}^{\ell}\right)\right)= \begin{cases}0 & t \notin\{i, q\} \\ X_{j}\left(b_{i}^{\ell}\right) & t=i \\ 0 & t=q\end{cases}
$$


and by Lemma $2.15 b_{i}^{\ell}=0$, so that $c_{t}^{k \ell}=0$ for all $t \in \bar{n}$. Finally

$$
\gamma_{p q}^{\ell} c_{t}^{\ell k}=\gamma_{p q}^{\ell} Y_{\ell}\left(a_{t}^{k}\right)= \begin{cases}0 & t \notin\{i, j\} \\ X_{q}\left(b_{i}^{k}\right) & t=i \\ 0 & t=j\end{cases}
$$

and by Lemma $2.15 b_{i}^{k}=0$, so that $c_{t}^{\ell k}=0$ for all $t \in \bar{n}$.

In the end we have that all $Y$-derivatives and all $X$-derivatives of $a_{t}^{k}$ are zero for all choices of the indices, so the system "closes up". Indeed, all the derivatives of the functions $g^{k}, f_{t}, \lambda_{t}, a_{t}^{k}, b_{t}^{k}$, for any $t \in \bar{n}$ and $k \in \bar{m}$ are expressed in terms of the functions themselves, as indicated by equations (5) through (11).

\subsection{2 - The connection.}

Recall that our strategy to prove rigidity is to introduce a connection on a vector bundle in such a way that finding a solution to the multicontact system is equivalent to finding a covariant constant section of the bundle. The bundle we are referring to is a trivial subbundle of the trivial $r$-plane bundle $\pi: T \rightarrow N$ over $N$, whose total space is $T=\mathbb{R}^{r}$, with $r=m+2 n+2 m n$. The idea is the following: once the multicontact structure has been selected, that is, the bases $\left\{X_{1}, \ldots, X_{n}\right\}$ and $\left\{Y_{1}, \ldots, Y_{m}\right\}$ have been fixed in such a way that assumptions (b) and (c) are satisfied, we choose only those directions along which the coordinates of a prolonged multicontact vector field are possibly non vanishing, as described by the lemmas of the previous sections. The fiber coordinates will thus be indexed by arrays of the form

$$
\left(\begin{array}{lll} 
& f & a \\
g & & \\
& \lambda & b
\end{array}\right)
$$

where, first of all, $f, \lambda \in \mathbb{R}^{n}, g \in \mathbb{R}^{m}$. As for the vectors $a$ and $b$, they will be labeled $a=\left(a_{i}^{k}\right)$ and $b=\left(b_{j}^{\ell}\right)$ respectively, with indices $(i, k)$ and $(j, \ell)$ running only in those index sets that correspond to non vanishing coordinates of multicontact vector fields. As already illustrated, the results of the previous section show that for each $k \in \bar{m}$ there exists a set $A_{k}$ (consisting at most of two indices) such that $a_{p}^{k}=0$ if $p \notin A_{k}$, whenever $a_{p}^{k}$ is the prolonged coordinate of a multicontact vector field. Notice that if $i, j \in A_{k}$ it may well be that $a_{i}^{k} \neq a_{j}^{k}$. We thus introduce the index set

$$
A=\left\{(i, k) \in \bar{n} \times \bar{m}: i \in A_{k}\right\}
$$

in order to parametrize the $a$-vector, that is we take $a=\left(a_{i}^{k}\right)$ with $(i, k) \in A$. 
Similarly, for each $\ell \in \bar{m}$ there exists a set $\tilde{B}_{\ell}$ (consisting at most of two indices) such that $b_{q}^{\ell}=0$ if $q \notin \tilde{B}_{\ell}$, whenever $b_{q}^{\ell}$ is the prolonged coordinate of a multicontact vector field. However, if $i \neq j$ are in $\tilde{B}_{\ell}$, then $b_{i}^{\ell}=b_{j}^{\ell}$. We thus define a new index set $B_{\ell}$ consisting at most of a single index in order to achieve a parametrization of the $b$-vector, by choosing one of $i$ or $j$. Finally, we introduce the index set

$$
B=\left\{(j, \ell) \in \bar{n} \times \bar{m}: j \in B_{\ell}\right\}
$$

and consider $b=\left(b_{j}^{\ell}\right)$ with $(j, \ell) \in B$. Hence $a \in \mathbb{R}^{c(A)}$ and $b \in \mathbb{R}^{c(B)}$, where $c(A)$ and $c(B)$ are the cardinalities of $A$ and $B$, respectively. The bundle we shall be concerned with is therefore $p: E \rightarrow N$ where the total space is $E=\mathbb{R}^{s}$, with $s=m+2 n+c(A)+c(B)$.

The sections $S \in \Gamma(E)$ of $E$ are mappings $S: N \rightarrow E$ such that $\pi \circ S=\mathrm{id}_{N}$ that we arrange in arrays as follows

$$
x \mapsto S(x)=\left(\begin{array}{ccc} 
& f(x) & a(x) \\
g(x) & \lambda(x) & b(x)
\end{array}\right) \in E
$$

where $g$ is a column vector in $\mathbb{R}^{m}, f, \lambda$ are s row vectors in $\mathbb{R}^{n}$ and $a \in \mathbb{R}^{c(A)}$ and $b \in \mathbb{R}^{c(B)}$ are vectors whose entries are arranged in (mostly empty) $n \times m$ matrices. For practical purposes, it is convenient to think of $E$ as a subbundle of $T$ and hence to regard $a$ and $b$ as $n \times m$ matrices whose entries are zero in the complement of the index sets $A$ and $B$. Omitting the dependence on $x$, one may thus think

$$
S=\left(\begin{array}{ccccccc} 
& & & & a_{1}^{1} & \cdots & a_{n}^{1} \\
& f_{1} & \cdots & f_{n} & \vdots & \ddots & \vdots \\
g^{1} & & & & a_{1}^{m} & \cdots & a_{n}^{m} \\
\vdots & & & & & & \\
g^{m} & & & & b_{1}^{1} & \ldots & b_{n}^{1} \\
& \lambda_{1} & \cdots & \lambda_{n} & \vdots & \ddots & \vdots \\
& & & & b_{1}^{m} & \cdots & b_{n}^{m}
\end{array}\right)
$$

and remember that most $a$ 's and $b$ 's are zero for $S \in \Gamma(E)$.

An affine connection on $N$ is an R-bilinear map $\nabla: \mathfrak{X}(N) \times \Gamma(E) \rightarrow$ $\rightarrow \Gamma(E)$, written $\nabla(X, S)=\nabla_{X} S$, with the following properties:

(i) $\nabla_{\varphi X} S=\varphi \nabla_{X} S$ for all $\varphi \in \mathcal{F}(N)$ all $X \in \mathfrak{X}(N)$ and all $S \in \Gamma(E)$;

(ii) $\nabla_{X}(\varphi S)=X(\varphi) S+\varphi \nabla_{X} S$ for all $\varphi \in \mathcal{F}(N)$, all $X \in \mathfrak{X}(N)$ and all $S \in \Gamma(E)$. 
We will define a connection on $E$ by specifying its values $\nabla_{X_{i}} S$ for all $i \in \bar{n}$ and $\nabla_{Y_{k}} S$ for all $k \in \bar{m}$ and then using (i). We shall do so by using the right hand sides of the various (affine) equations that have been obtained in the previous section. In order to write explicitly the definition, we parametrize $S$ as in (12). Instead of writing $\nabla_{X_{i}} S$ and $\nabla_{Y_{k}} S$ as large arrays, we introduce the dual bases $\left(g^{s}\right)^{*}, f_{t}^{*}, \lambda_{t}^{*},\left(a_{t}^{S}\right)^{*}$ and $\left(b_{t}^{s}\right)^{*}$. Here is the definition of the various components of $\nabla_{Y_{k}} S$ :

$$
\begin{aligned}
& \left(g^{s}\right)^{*}\left(\nabla_{Y_{k}} S\right)=Y_{k}\left(g^{s}\right)+\delta_{k s}\left(\lambda_{i}+\lambda_{j}\right) \\
& f_{t}^{*}\left(\nabla_{Y_{k}} S\right)= \begin{cases}Y_{k}\left(f_{t}\right)-a_{t}^{k} & \text { if }(t, k) \in A \\
Y_{k}\left(f_{t}\right) & \text { if }(t, k) \notin A\end{cases} \\
& \lambda_{t}^{*}\left(\nabla_{Y_{k}} S\right)= \begin{cases}Y_{k}\left(\lambda_{t}\right)-b_{t}^{k} & \text { if }(t, k) \in B \\
Y_{k}\left(\lambda_{t}\right) & \text { if }(t, k) \notin B\end{cases} \\
& \left(a_{t}^{s}\right)^{*}\left(\nabla_{Y_{k}} S\right)=Y_{k}\left(a_{t}^{s}\right) \\
& \left(b_{t}^{s}\right)^{*}\left(\nabla_{Y_{k}} S\right)=Y_{k}\left(b_{t}^{s}\right) .
\end{aligned}
$$

For the sake of precision we remark that in the first line one picks any pair $(i, j)$ with the property that $\left[X_{i}, X_{j}\right]=\gamma_{i j}^{k} Y_{k}$ (with $\gamma_{i j}^{k} \neq 0$ ), certainly existing by our hypotheses, and the sum $\lambda_{i}+\lambda_{j}$ is independent of such choice; further, the last two lines are defined for $(t, s)$ in $A$ and $B$, respectively. Similarly, the definition of the various components of $\nabla_{X_{i}} S$ is:

$$
\begin{aligned}
& \left(g^{s}\right)^{*}\left(\nabla_{X_{i}} S\right)=X_{i}\left(g^{s}\right)-\sum_{t \in \bar{n}} \alpha_{t i}^{s} f_{t} \\
& f_{t}^{*}\left(\nabla_{X_{i}} S\right)=X_{i}\left(f_{t}\right)+\delta_{i t} \lambda_{t} \\
& \lambda_{t}^{*}\left(\nabla_{X_{i}} S\right)= \begin{cases}X_{i}\left(\lambda_{t}\right)-\sum_{(t, \ell) \in A} \alpha_{t i}^{\ell} a_{t}^{\ell} & \text { if } t \neq i \\
X_{i}\left(\lambda_{i}\right)-2 \gamma_{i j}^{k} a_{j}^{k} & \text { if } t=i \text { and }(j, k) \in A \\
X_{i}\left(\lambda_{i}\right) & \text { if } t=i \text { and }(j, k) \notin A\end{cases} \\
& \left(a_{t}^{s}\right)^{*}\left(\nabla_{X_{i}} S\right)=X_{i}\left(a_{t}^{s}\right)+\delta_{i t} b_{t}^{s} \\
& \left(b_{t}^{s}\right)^{*}\left(\nabla_{X_{i}} S\right)=X_{i}\left(b_{t}^{s}\right) .
\end{aligned}
$$

Here again the last two lines are defined for $(t, s)$ in $A$ and $B$, respectively; as for the value of $\lambda_{t}^{*}\left(\nabla_{X_{i}} S\right)$, the index $j$ appearing in the right hand side is an index such that $\left[X_{i}, X_{j}\right]=\gamma_{i j}^{k} Y_{k}$ (with $\gamma_{i j}^{k} \neq 0$ ), certainly existing by our hypotheses. 
Proposition 2.17. Formulae (13) and (14) define an affine connection on $E$.

Proof. Let $X$ be any vector field in the basis and let $S$ be a section. We temporarily change notation and write $S$ as a vector with components $S_{i}$ with $i$ ranging in the index set $I$ and observe that formulae (13) and (14) are all of the form

$$
\left(\nabla_{X}(S)\right)_{i}=X\left(S_{i}\right)+\sum_{j \in I} C_{j} S_{k(j)}
$$

where $j \mapsto k(j)$ is some mapping of $I$ and $C_{j}$ is a possibly zero constant. Property (i) is automatic from our definition. As for (ii), for any smooth $\psi$ we have

$$
\begin{aligned}
\left(\nabla_{X}(\psi S)\right)_{i} & =X\left(\psi S_{i}\right)+\sum_{j \in I} C_{j} \psi S_{k(j)} \\
& =X(\psi) S_{i}+\psi X\left(S_{i}\right)+\sum_{j \in I} C_{j} \psi S_{k(j)} \\
& =X(\psi) S_{i}+\psi\left(\nabla_{X}(S)\right)_{i},
\end{aligned}
$$

as desired.

\subsection{3 - End of proof.}

Summarizing, the vector field (3) is a multicontact vector field if and only if the functions $\left\{f_{t}, \lambda_{t}, g^{s}, a_{t}^{s}, b_{t}^{s}: t \in \bar{n}, s \in \bar{m}\right\}$ satisfy the equations (5) through (11) and this in turn happens if and only if the section $S$ defined by (12) is a covariant constant section of the bundle $p: E \rightarrow N$ with respect to the connection $\nabla$, that is, if it satisfies $\nabla_{X} S=0$ for any $X \in \mathfrak{X}(N)$. Recall that the bundle we shall be concerned with is therefore $p: E \rightarrow N$ where the total space is $E=\mathbb{R}^{s}$, with $s=m+2 n+c(A)+c(B)$. Thus, there are at most $s$ linearly independent such sections, and this proves that the space of multicontact vector fields is at most $s$-dimensional.

There are no a priori conditions on the $f, \lambda \in \mathbb{R}^{n}, g \in \mathbb{R}^{m}$. Therefore, they may contribute up to $2 n+m$ dimensions. Moreover, we consider $a=\left(a_{i}^{k}\right)$ with $(i, k) \in A$ and $b=\left(b_{j}^{\ell}\right)$ with $(j, \ell) \in B$. Hence $a \in \mathbb{R}^{c(A)}$ and $b \in \mathbb{R}^{c(B)}$, where $c(A)$ and $c(B)$ are the cardinalities of $A$ and $B$, respectively. We achieve the total number of $s$.

In the next section we improve our result by proving that the connection $\nabla$ is actually flat. 


\section{Flatness.}

The curvature operator $R(X, Y): \Gamma(E) \rightarrow \Gamma(E)$ associated to $\nabla$ is of course

$$
R(X, Y) S=\nabla_{X} \nabla_{Y} S-\nabla_{Y} \nabla_{X} S-\nabla_{[X, Y]} S
$$

for any $X, Y \in \mathfrak{X}(N)$ and any $S \in \Gamma(E)$. Evidently, $R$ is known whenever $R\left(Y_{k}, Y_{\ell}\right), R\left(X_{i}, Y_{k}\right)$ and $R\left(X_{i}, X_{j}\right)$ are known, for any $k, \ell \in \bar{m}$ and $i, j \in \bar{n}$.

The intricacy of some of the computations that follow calls for some notational simplification. In particular, we shall adopt a slight abuse of notation and, instead of the precise version appearing in formula (13) that distinguishes among pairs $(t, k) \in A$ and pairs $(t, k) \notin A$, we shall write

$$
f_{t}^{*}\left(\nabla_{Y_{k}} S\right)=Y_{k}\left(f_{t}\right)-a_{t}^{k}
$$

for all pairs $(t, k)$; the understanding is that $a_{t}^{k}=0$ if $(t, k) \notin A$. The same kind of convention is used for the definition of $\lambda_{t}^{*}\left(\nabla_{Y_{k}} S\right)$ and $\lambda_{t}^{*}\left(\nabla_{X_{i}} S\right)$.

Finally, we stress that the combinatorial structure of the sets $A$ and $B$ is described by the various Lemmas of the previous section. We shall therefore appeal to them when needed, with the understanding that if the Lemma states that some jet variable $a_{i}^{k}$ (of a prolonged multicontact vector field) vanishes in a certain configuration, then evidently $(i, k) \notin A$ in the same configuration, and similarly for $B$.

Lemma 3.1. $R\left(Y_{k}, Y_{\ell}\right) S=0$ for all $k, \ell \in \bar{m}$.

Proof. Since $\left[Y_{k}, Y_{\ell}\right]=0$ for any $k, \ell \in \bar{m}, R\left(Y_{k}, Y_{\ell}\right) S=\nabla_{Y_{k}} \nabla_{Y_{\ell}} S-$ $-\nabla_{Y_{\ell}} \nabla_{Y_{k}} S$. By (13) and by symmetry, a straightforward calculation shows that

$$
f_{t}^{*}\left(R\left(Y_{k}, Y_{\ell}\right) S\right)=\lambda_{t}^{*}\left(R\left(Y_{k}, Y_{\ell}\right) S\right)=0
$$

for all $t \in \bar{n}$ and

$$
\left(a_{t}^{s}\right)^{*}\left(R\left(Y_{k}, Y_{\ell}\right) S\right)=0, \quad\left(b_{t}^{s}\right)^{*}\left(R\left(Y_{k}, Y_{\ell}\right) S\right)=0
$$

for all $(t, s)$ in $A$ and $B$, respectively. Let $\ell, k \in \bar{m}$ with $\ell \neq k$. By assumption (b) we may choose $i, j, p, q \in \bar{n}$ such that $\left[X_{i}, X_{j}\right]=\gamma_{i j}^{k} Y_{k}$ and $\left[X_{p}, X_{q}\right]=$ $=\gamma_{p q}^{\ell} Y_{\ell}$. By (13) we obtain that for every $s \in \bar{m}$

$$
\begin{aligned}
& \left(g^{s}\right)^{*}\left(\nabla_{Y_{k}} \nabla_{Y_{\ell}} S\right)=Y_{k}\left(Y_{\ell}\left(g^{s}\right)+\delta_{\ell s}\left(\lambda_{p}+\lambda_{q}\right)\right)+\delta_{k s}\left(Y_{\ell}\left(\lambda_{i}\right)-b_{i}^{\ell}+Y_{\ell}\left(\lambda_{j}\right)-b_{j}^{\ell}\right) \\
& \left(g^{s}\right)^{*}\left(\nabla_{Y_{\ell}} \nabla_{Y_{k}} S\right)=Y_{\ell}\left(Y_{k}\left(g^{s}\right)+\delta_{k s}\left(\lambda_{i}+\lambda_{j}\right)\right)+\delta_{\ell s}\left(Y_{k}\left(\lambda_{p}\right)-b_{p}^{k}+Y_{k}\left(\lambda_{q}\right)-b_{q}^{k}\right) .
\end{aligned}
$$


Therefore, recalling that all the $Y$ 's commute and subtracting the previous formulae

$$
\left(g^{s}\right)^{*}\left(R\left(Y_{k}, Y_{\ell}\right) S\right)= \begin{cases}b_{p}^{k}+b_{q}^{k} & \text { if } s=\ell \\ -b_{i}^{\ell}-b_{j}^{\ell} & \text { if } s=k \\ 0 & \text { if } s \notin\{\ell, k\} .\end{cases}
$$

In all cases $(p, k),(q, k) \notin B$ and $(i, \ell),(j, \ell) \notin B$. Indeed, this is very clear when $\{p, q\}$ and $\{i, j\}$ are disjoint pairs and follows from Lemma 2.15 when they are not. Hence $\left(g^{s}\right)^{*}\left(R\left(Y_{k}, Y_{\ell}\right) S\right)=0$, as desired.

Lemma 3.2. $\quad R\left(X_{i}, Y_{k}\right) S=0$ for all $i \in \bar{n}$ and all $k \in \bar{m}$.

Proof. Since $\left[X_{i}, Y_{k}\right]=0, R\left(X_{i}, Y_{k}\right) S=\nabla_{X_{i}} \nabla_{Y_{k}} S-\nabla_{Y_{k}} \nabla_{X_{i}} S$ for any $i \in \bar{n}, k \in \bar{m}$. By (13) and (14), using again $\left[X_{i}, Y_{k}\right]=0$, a straightforward calculation shows that

$$
f_{t}^{*}\left(R\left(X_{i}, Y_{k}\right) S\right)=0, \quad\left(a_{t}^{s}\right)^{*}\left(R\left(X_{i}, Y_{k}\right) S\right)=0, \quad\left(b_{t}^{s}\right)^{*}\left(R\left(X_{i}, Y_{k}\right) S\right)=0
$$

for all $t \in \bar{n}$ and for all $(t, s) \in A$ and $B$, respectively.

In order to show that for any given $i \in \bar{n}$ and $k \in \bar{m}$ we have $\lambda_{t}^{*}\left(R\left(X_{i}, Y_{k}\right) S\right)=0$ for all $t \in \bar{n}$ and $\left(g^{s}\right)^{*}\left(R\left(X_{i}, Y_{k}\right) S\right)=0$ for all $s \in \bar{m}$, we must distinguish between the following possibilities:

(i) there exists $j \in \bar{n}$ such that $\left[X_{i}, X_{j}\right]=\gamma_{i j}^{k} Y_{k}$;

(ii) for no $j \in \bar{n}$ we have $\left[X_{i}, X_{j}\right]=\gamma_{i j}^{k} Y_{k}$ and hence, by assumption (c), we can find $\kappa \in \bar{m}$, with $\kappa \neq k$, and $j \in \bar{n}$ such that $\left[X_{i}, X_{j}\right]=\gamma_{i j}^{\kappa} Y_{\kappa}$.

In case (i), by (13) and (14) we have

$$
\lambda_{t}^{*}\left(\nabla_{X_{i}} \nabla_{Y_{k}} S\right)= \begin{cases}X_{i}\left(Y_{k}\left(\lambda_{t}\right)-b_{t}^{k}\right)-\sum_{(t, \ell) \in A} \alpha_{t i}^{\ell} Y_{k}\left(a_{t}^{\ell}\right) & \text { if } t \neq i \\ X_{i}\left(Y_{k}\left(\lambda_{i}\right)-b_{i}^{k}\right)-2 \gamma_{i j}^{k} Y_{k}\left(a_{j}^{k}\right) & \text { if } t=i\end{cases}
$$

and

$$
\lambda_{t}^{*}\left(\nabla_{Y_{k}} \nabla_{X_{i}} S\right)= \begin{cases}Y_{k}\left(X_{i}\left(\lambda_{t}\right)-\sum_{(t, \ell) \in A} \alpha_{t i}^{\ell} a_{t}^{\ell}\right)-X_{i}\left(b_{t}^{k}\right) & \text { if } t \neq i \\ Y_{k}\left(X_{i}\left(\lambda_{i}\right)-2 \gamma_{i j}^{k} a_{j}^{k}\right)-X_{i}\left(b_{i}^{k}\right) & \text { if } t=i .\end{cases}
$$

Since $\left[X_{i}, Y_{k}\right]=0$, by subtraction we get $\lambda_{t}^{*}\left(R\left(X_{i}, Y_{k}\right) S\right)=0$. Next, by (13) 
and (14)

$$
\begin{aligned}
& \left(g^{s}\right)^{*}\left(\nabla_{X_{i}} \nabla_{Y_{k}} S\right)=X_{i}\left(Y_{k}\left(g^{s}\right)+\delta_{k s}\left(\lambda_{i}+\lambda_{j}\right)\right)-\sum_{t \in \bar{n}} \alpha_{t i}^{s}\left(Y_{k}\left(f_{t}\right)-a_{t}^{k}\right) \\
& \left(g^{s}\right)^{*}\left(\nabla_{Y_{k}} \nabla_{X_{i}} S\right)=Y_{k}\left(X_{i}\left(g^{s}\right)-\sum_{t \in \bar{n}} \alpha_{t i}^{s} f_{t}\right)+\delta_{k s}\left(X_{i}\left(\lambda_{j}\right)+\gamma_{i j}^{k} a_{j}^{k}+X_{i}\left(\lambda_{i}\right)-2 \gamma_{i j}^{k} a_{j}^{k}\right) .
\end{aligned}
$$

Once again, $\left[X_{i}, Y_{k}\right]=0$ and subtraction yield

$$
\left(g^{s}\right)^{*}\left(R\left(X_{i}, Y_{k}\right) S\right)=\sum_{t \in \bar{n}} \alpha_{t i}^{s} a_{t}^{k}+\delta_{k s}\left(\gamma_{i j}^{k} a_{j}^{k}\right)
$$

If $s \neq k$, by item (i) of Lemma 2.8, the sum on the right hand side vanishes. If $s=k$, by Lemma $2.7(t, k) \notin A$ whenever $t \notin\{i, j\}$ and hence

$$
\sum_{t \in \bar{n}} \alpha_{t i}^{k} a_{t}^{k}+\gamma_{i j}^{k} a_{j}^{k}=-\gamma_{i j}^{k} a_{j}^{k}+\gamma_{i j}^{k} a_{j}^{k}=0 .
$$

In case (ii), $i \in \bar{n}$ and $k \in \bar{m}$ are such that for no $j \in \bar{n}$ we have $\left[X_{i}, X_{j}\right]=\gamma_{i j}^{k} Y_{k}$. Our assumptions come now into the picture as follows: by assumption (b) we can find $i_{k}, j_{k} \in \bar{n}$ such that $\left[X_{i_{k}}, X_{j_{k}}\right]=\gamma_{i_{k} j_{k}}^{k} Y_{k}$ where $i \notin\left\{i_{k}, j_{k}\right\}$; furthemore, by assumption (c) we can find $\kappa \in \bar{m}$ with $\kappa \neq k$ and $j \in \bar{n}$ such that $\left[X_{i}, X_{j}\right]=\gamma_{i j}^{\kappa} Y_{\kappa}$.

Now, using again (13) and (14) we obtain equations analogous to (15) and (16), but with $\kappa$ in place of $k$, and we conclude that $\lambda_{t}^{*}\left(R\left(X_{i}, Y_{k}\right) S\right)=0$. Finally, using one last time (13) and (14) we get

$$
\begin{aligned}
\left(g^{s}\right)^{*}\left(\nabla_{X_{i}} \nabla_{Y_{k}} S\right) & =X_{i}\left(Y_{k}\left(g^{s}\right)+\delta_{k s}\left(\lambda_{i_{k}}+\lambda_{j_{k}}\right)\right)-\sum_{t \in \bar{n}} \alpha_{t i}^{s}\left(Y_{k}\left(f_{t}\right)-a_{t}^{k}\right) \\
\left(g^{s}\right)^{*}\left(\nabla_{Y_{k}} \nabla_{X_{i}} S\right) & =Y_{k}\left(X_{i}\left(g^{s}\right)-\sum_{t \in \bar{n}} \alpha_{t i}^{s} f_{t}\right) \\
& +\delta_{k s}\left(X_{i}\left(\lambda_{i_{k}}\right)-\sum_{\left(i_{k}, \ell\right) \in A} \alpha_{i_{k} i}^{\ell} a_{i_{k}}^{\ell}+X_{i}\left(\lambda_{j_{k}}\right)-\sum_{\left(i_{k}, \ell\right) \in A} \alpha_{j_{k} i}^{\ell} a_{j_{k}}^{\ell}\right) .
\end{aligned}
$$

Therefore, since $\left[X_{i}, Y_{k}\right]=0$, we subtract the two previous equalities and obtain

$$
\left(g^{s}\right)^{*}\left(R\left(X_{i}, Y_{k}\right) S\right)=\sum_{t \in \bar{n}} \alpha_{t i}^{s} a_{t}^{k}+\delta_{k s}\left(\sum_{\left(i_{k}, \ell\right) \in A} \alpha_{i_{k} i}^{\ell} a_{i_{k}}^{\ell}+\sum_{\left(i_{k}, \ell\right) \in A} \alpha_{j_{k}}^{\ell} a_{j_{k}}^{\ell}\right) .
$$

If $s \neq k$, by item (i) of Lemma 2.8, the sum on the right hand side vanishes. If $s=k$, by Lemma $2.7(t, k) \notin A$ whenever $t \notin\left\{i_{k}, j_{k}\right\}$. Hence

$$
\left(g^{k}\right)^{*}\left(R\left(X_{i}, Y_{k}\right) S\right)=2\left(\alpha_{i_{k} i}^{k} a_{i_{k}}^{k}+\alpha_{j_{k}}^{k} a_{j_{k}}^{k}\right)+\sum_{\left(i_{k}, \ell\right) \in A, \ell \neq k} \alpha_{i_{k} i}^{\ell} a_{i_{k}}^{\ell}+\sum_{\left(j_{k}, \ell\right) \in A, \ell \neq k} \alpha_{j_{k} i}^{\ell} a_{j_{k}}^{\ell} .
$$


By Lemma 2.9, if $\alpha_{i_{k} i}^{k} \neq 0$ then $\left(i_{k}, k\right) \notin A$ because $i \neq j_{k}$. Similarly, if $\alpha_{j_{k} i}^{k} \neq 0$ we have that $\left(j_{k}, k\right) \notin A$ and so

$$
\left(g^{k}\right)^{*}\left(R\left(X_{i}, Y_{k}\right) S\right)=\sum_{\left(i_{k}, \ell\right) \in A, \ell \neq k} \alpha_{i_{k}}^{\ell} a_{i_{k}}^{\ell}+\sum_{\left(j_{k}, \ell\right) \in A, \ell \neq k} \alpha_{j_{k}}^{\ell} a_{j_{k}}^{\ell} .
$$

We show now that for all indices $\ell \in \bar{m}$ with $\ell \neq k$, the sum on the right hand side vanishes, whence the result. Fix any such $\ell$. We prove that $\alpha_{i_{k}}^{\ell} a_{i_{k}}^{\ell}=0$. By Lemma 2.6, $\left(i_{k}, \ell\right) \in A$ only if there exists $p \in \bar{n}$ such that $\left[X_{i_{k}}, X_{p}\right]=\gamma_{i_{k} p}^{\ell} Y_{\ell}$. Suppose that $\alpha_{i_{k} i}^{\ell} \neq 0$, for otherwise $\alpha_{i_{k} i}^{\ell} a_{i_{k}}^{\ell}=0$. By Lemma 2.9 , if $p \neq i$ we have that $\left(i_{k}, \ell\right) \notin A$. If $p=i$, that is $\left[X_{i_{k}}, X_{i}\right]=$ $=\gamma_{i_{k} i} Y_{\ell}$ by Lemma $2.10\left(i_{k}, \ell\right) \notin A$ for any $\ell \in \bar{m}$. An analogous argument shows that $\alpha_{j_{k}}^{\ell} a_{j_{k}}^{\ell}=0$ for any $\ell \in \bar{m}$. Hence $\left(g^{s}\right)^{*}\left(R\left(X_{i}, Y_{k}\right) S\right)=0$ for all $s \in \bar{m}$.

Lemma 3.3. $R\left(X_{i}, X_{j}\right) S=0$ for all $i, j \in \bar{n}$.

Proof. Clearly, $R\left(X_{i}, X_{j}\right) S=\nabla_{X_{i}} \nabla_{X_{j}} S-\nabla_{X_{j}} \nabla_{X_{i}} S-\nabla_{\left[X_{i}, X_{j}\right]} S$ for any $i, j \in \bar{n}$. The tensoriality in $X$ of $\nabla_{X} S$ and (13) give

$$
\begin{aligned}
& \left(a_{t}^{s}\right)^{*}\left(\nabla_{\left[X_{i}, X_{j}\right]} S\right)=\left(a_{t}^{s}\right)^{*}\left(\nabla_{\sum_{\ell \in \bar{m}} \alpha_{i j}^{\ell} Y_{\ell}} S\right)=\sum_{\ell \in \bar{m}} \alpha_{i j}^{\ell} Y_{\ell}\left(a_{t}^{s}\right) \\
& \left(b_{t}^{s}\right)^{*}\left(\nabla_{\left[X_{i}, X_{j}\right]} S\right)=\left(b_{t}^{s}\right)^{*}\left(\nabla_{\sum_{\ell \in \bar{m}} \alpha_{i j}^{\ell} Y_{\ell}} S\right)=\sum_{\ell \in \bar{m}} \alpha_{i j}^{\ell} Y_{\ell}\left(b_{t}^{s}\right) .
\end{aligned}
$$

Using (14) and symmetry, a straightforward calculation shows that

$$
\left(a_{t}^{S}\right)^{*}\left(R\left(\nabla_{X_{i}} \nabla_{X_{j}}\right) S\right)=0 \quad\left(b_{t}^{s}\right)^{*}\left(R\left(\nabla_{X_{i}} \nabla_{X_{j}}\right) S\right)=0
$$

for all $(t, s) \in A$ and $B$, respectively.

Next (14) yields

$$
\left(g^{s}\right)^{*}\left(\nabla_{X_{i}} \nabla_{X_{j}} S\right)=X_{i}\left(X_{j}\left(g^{s}\right)-\sum_{t \in \bar{n}} \alpha_{t j}^{s} f_{t}\right)-\sum_{t \in \bar{n}} \alpha_{t i}^{s}\left(X_{j}\left(f_{t}\right)+\delta_{j t} \lambda_{t}\right) .
$$

By (b), for all $s \in \bar{m}$, there exists $i_{s}, j_{s} \in \bar{n}$ such that $\left[X_{i_{s}}, X_{j_{s}}\right]=\gamma_{i_{s} j_{s}}^{s} Y_{s}$ and by (13)

$$
\begin{aligned}
\left(g^{s}\right)^{*}\left(\nabla_{\left[X_{i}, X_{j}\right]} S\right) & =\sum_{\ell \in \bar{m}} \alpha_{i j}^{\ell}\left(Y_{\ell}\left(g^{s}\right)+\delta_{\ell s}\left(\lambda_{i_{s}}+\lambda_{j_{s}}\right)\right) \\
& =\sum_{\ell \in \bar{m}} \alpha_{i j}^{\ell} Y_{\ell}\left(g^{s}\right)+\alpha_{i j}^{s}\left(\lambda_{i_{s}}+\lambda_{j_{s}}\right) .
\end{aligned}
$$


A straightforward calculation gives

$$
\left(g^{s}\right)^{*}\left(R\left(X_{i}, X_{j}\right) S\right)=\alpha_{i j}^{s}\left(\lambda_{i}+\lambda_{j}\right)-\alpha_{i j}^{s}\left(\lambda_{i_{s}}+\lambda_{j_{s}}\right) .
$$

By Corollary 2.5, for all $s$ such that $\alpha_{i j}^{s} \neq 0$ we have that $\lambda_{i}+\lambda_{j}=\lambda_{i_{s}}+\lambda_{j_{s}}$, therefore $\left(g^{s}\right)^{*}\left(R\left(X_{i}, X_{j}\right) S\right)=0$.

Next, by (13), (14) and symmetry we obtain that

$$
f_{t}^{*}\left(R\left(X_{i}, X_{j}\right) S\right)= \begin{cases}\sum_{\ell \in \bar{m}} \alpha_{i j}^{\ell} a_{t}^{\ell} & \text { if } t \notin\{i, j\} \\ 0 & \text { if } t \in\{i, j\} .\end{cases}
$$

By Lemma 2.7, the sum on the first line vanishes; so $f_{t}^{*}\left(R\left(X_{i}, X_{j}\right) S\right)=0$.

We are left to show that for any given $t \in \bar{n}$ we have that $\lambda_{t}^{*}\left(R\left(X_{i}, X_{j}\right) S\right)=0$.

By assumption (b) there exist $\mu, v \in \bar{n}$ and $k, \kappa \in \bar{m}$ such that $\left[X_{i}, X_{\mu}\right]=\gamma_{i \mu}^{k} Y_{k}$ and $\left[X_{j}, X_{v}\right]=\gamma_{j v}^{\kappa} Y_{\kappa}$. By (13) and (14) we obtain

$$
\lambda_{t}^{*}\left(\nabla_{X_{i}} \nabla_{X_{j}} S\right)= \begin{cases}X_{i}\left(X_{j}\left(\lambda_{t}\right)-\sum_{(t, \ell) \in A} \alpha_{t j}^{\ell} a_{t}^{\ell}\right)-\sum_{(t, \ell) \in A} \alpha_{t i}^{\ell}\left(X_{j}\left(a_{t}^{\ell}\right)+\delta_{j t} b_{t}^{\ell}\right) & \text { if } t \notin\{i, j\} \\ X_{i}\left(X_{j}\left(\lambda_{j}\right)-2 \gamma_{j v}^{\kappa} a_{v}^{\kappa}\right)-\sum_{(j, \ell) \in A} \alpha_{j i}^{\ell}\left(X_{j}\left(a_{j}^{\ell}\right)+b_{j}^{\ell}\right) & \text { if } t=j \\ X_{i}\left(X_{j}\left(\lambda_{i}\right)-\sum_{(i, \ell) \in A} \alpha_{i j}^{\ell} a_{i}^{\ell}\right)-2 \gamma_{i \mu}^{k}\left(X_{j}\left(a_{\mu}^{k}\right)+\delta_{j \mu} b_{\mu}^{k}\right) & \text { if } t=i .\end{cases}
$$

A straightforward computation, gives

$$
\lambda_{t}^{*}\left(R\left(X_{i}, X_{j}\right) S\right)= \begin{cases}\sum_{\ell \in \bar{m}} \alpha_{i j}^{\ell} b_{t}^{\ell} & \text { if } t \notin\{i, j\} \\ 2 \gamma_{j v}^{\kappa} \delta_{i v} b_{v}^{\kappa}+2 \sum_{(j, \ell) \in B} \alpha_{i j}^{\ell} b_{j}^{\ell} & \text { if } t=j \\ -2 \gamma_{i \mu}^{k} \delta_{j \mu} b_{\mu}^{k}+2 \sum_{(i, \ell) \in B} \alpha_{i j}^{\ell} b_{i}^{\ell} & \text { if } t=i .\end{cases}
$$

By Lemma 2.13, the first line vanishes. We show now that for all $\ell \in \bar{m}$, the sums on the second and third lines vanish, whence the result. Two cases arise: either $j=\mu$ or $j \neq \mu$. In the first case, $\alpha_{i j}^{k}=\gamma_{i \mu}^{k}$ and $\alpha_{i j}^{\ell}=0$ for any $\ell \in \bar{m}$ with $\ell \neq k$, so

$$
\lambda_{t}^{*}\left(R\left(\nabla_{X_{i}} \nabla_{X_{j}}\right) S\right)= \begin{cases}0 & \text { if } t \notin\{i, j\} \\ 2 \gamma_{j v}^{k} \delta_{i v} b_{v}^{\kappa}+2 \gamma_{i \mu}^{k} b_{j}^{k} & \text { if } t=j \\ -2 \gamma_{i \mu}^{k} b_{j}^{k}+2 \gamma_{i \mu}^{k} b_{i}^{k} & \text { if } t=i .\end{cases}
$$


By Lemma $2.12 b_{i}^{k}=b_{j}^{k}$, thus the third line vanishes. Next, if $i=v$ then $\gamma_{j v}^{\kappa}=-\gamma_{i \mu}^{k}, \kappa=k$ and by Lemma 2.12 we obtain

$$
2 \gamma_{j v}^{k} \delta_{i v} b_{v}^{\kappa}+2 \gamma_{i \mu}^{k} b_{j}^{k}=-2 \gamma_{i \mu}^{k} b_{i}^{k}+2 \gamma_{i \mu}^{k} b_{j}^{k}=0 .
$$

Otherwise, if $i \neq v$, by Lemma $2.15(j, k) \notin B$.

In the second case, $j \neq \mu$ (clearly $i \neq v$, for otherwise we obtain the configuration just discussed). We rewrite

$$
\lambda_{t}^{*}\left(R\left(X_{i}, X_{j}\right) S\right)= \begin{cases}0 & \text { if } t \notin\{i, j\} \\ 2 \alpha_{i j}^{\kappa} b_{j}^{\kappa}+2 \alpha_{i j}^{k} b_{j}^{k}+2 \sum_{(j, \ell) \in B, \ell \notin\{k, \kappa\}} \alpha_{i j}^{\ell} b_{j}^{\ell} & \text { if } t=j \\ 2 \alpha_{i j}^{\kappa} b_{i}^{\kappa}+2 \alpha_{i j}^{k} b_{i}^{k}+2 \sum_{(i, \ell) \in B, \ell \notin\{k, \kappa\}} \alpha_{i j}^{\ell} b_{i}^{\ell} & \text { if } t=i .\end{cases}
$$

By Lemma 2.14, if $\alpha_{i j}^{\kappa} \neq 0$ then $(j, \kappa) \notin B$. Similarly, if $\alpha_{i j}^{k} \neq 0$ then $(i, \kappa) \notin B$. Next, suppose that exists $\ell \in \bar{m}$ with $\ell \notin\{k, \kappa\}$ such that $\alpha_{i j}^{\ell} \neq 0$, for otherwise the sum on the right hand side vanishes. Fix any such $\ell$ : by assumption (b) there exists $p, q \in \bar{n}$ such that $\left[X_{p}, X_{q}\right]=\gamma_{p q}^{\ell} Y_{\ell}$ and two cases arise: either $\{i, j\} \cap\{p, q\}=\emptyset$ or, by symmetry we may assume, $p=j$. In the first case, by Lemma $2.13(i, \ell) \notin B$ and $(j, \ell) \notin B$. In the second case, by Lemma 2.13 and by Lemma $2.14(i, \ell) \notin B$ and $(j, \ell) \notin B$ respectively. Thus the second line vanishes and an analogous argument works for the third line. Therefore $\lambda_{t}^{*}\left(R\left(X_{i}, X_{j}\right) S\right)=0$.

We have proved:

THEOREM 3.4. The connection $\nabla$ introduced in Section 2.3.2 is always flat.

As a consequence, the dimension of Lie algebra $\mathcal{M}$ of multicontact vector fields is always equal to the dimension of the bundle, because flatness implies the existence of a maximal number of covariant constant sections. This allows simple dimensional computations.

\section{$3.1-$ Examples.}

We now look at some of the examples considered in Section 2.1.1 and either compute explicitly the dimension of the Lie algebra $\mathcal{M}$ of multicontact vector fields, or we give upper bounds. 


\subsection{1 - H-type algebras.}

Consider first the Heisenberg algebra, that is, let $\left\{X_{1}, \ldots, X_{2 p}\right\}$ be a basis of $\mathfrak{n}_{1}$ and let $\left\{T=Y_{1}\right\}$ be a basis of $\mathfrak{n}_{2}$. If $\left[X_{i}, X_{i+p}\right]=T$ and $\left[X_{i}, X_{j}\right]=0$ in all other cases we obtain of course the Heisenberg algebra. The dimension of the Lie algebra $\mathcal{M}$ of multicontact vector fields is $3 p+2$. Indeed, by Lemma 2.7 and by Lemma $2.13 \quad a_{i}^{1}=b_{i}^{1}=0$ for all $i \in\{1, \ldots, 2 p\}$. Next, by item (i) of Corollary 2.5 , we obtain that

$$
\lambda_{1}+\lambda_{p+1}=\lambda_{i}+\lambda_{p+i}
$$

for any $i \geq 2$; so $\lambda_{p+i} \in \operatorname{sp}\left\{\lambda_{1}, \ldots, \lambda_{p+1}\right\}$ for any $i \geq 2$. The functions $f_{t}$ for all $t \in\{1, \ldots, 2 p\}, \lambda_{s}$ for all $s \in\{1, \ldots, p+1\}$ and $g$ may be linearly indipendent and the total count is exactly $3 p+2$, as desired.

Look now at any H-type algebra and follow the notation of Section 2.1.1. Arguing as we just did, $\lambda_{p+i} \in \operatorname{sp}\left\{\lambda_{1}, \ldots, \lambda_{p+1}\right\}$ for any $i \geq 2$ and also $a_{i}^{1}=0$ and $b_{i}^{1}=0$, for all $i \in\{1, \ldots, 2 p\}$. Fix $k \in\{2, \ldots, m\}$, by Lemma $2.10 a_{i}^{k}$ vanish, and hence by Lemma $2.11, b_{i}^{k}=0$. Indeed, every $Y_{k}$ with $k \neq 1$ is of the form $\left[X_{i}, X_{j}\right]$ and ad $X_{i}$ sends either $X_{i+p}$ or $X_{i-p}$ to $\pm Y_{1}$, so that Lemma 2.10 implies $a_{i}^{k}=0$ and therefore $a_{q}^{k}$ vanish for all $(q, k) \in \bar{n} \times \bar{m}$; similarly for $j$. The functions $f_{t}$ and $g^{k}$ for all $t \in\{1, \ldots, 2 p\}$ and $k \in\{1, \ldots, m\}$ may be linearly independent and $\lambda_{1}, \ldots, \lambda_{p+1}$ is a maximal number of linearly independent elements among the $\lambda_{i}$. The total count is less then or equal to $3 p+1+m$. The dimensional bound is thus sharp.

\subsection{2 - Hessenberg algebras.}

Consider now the Hessenberg algebra $\mathfrak{n}^{(2)}$ associated to $\mathfrak{s}(n, \mathbb{R})$. For the case $n=3$ it was shown by Michael Eastwood [14] that the $\operatorname{dim} \mathcal{M}$ is eight. Incidentally, this is just the previous case for $p=2$. Now, we show that the dimension of the Lie algebra $\mathcal{M}$ of multicontact vector fields is 9 if $n=4$ and $3 n-4$ if $n>4$, in agreement with [16]. The brackets are as in (1).

By Lemma 2.15 we obtain that $b_{i}^{k}=0$ for all $i \in\{1, \ldots, n-1\}, k \in$ $\in\{1, \ldots, n-2\}$. By Lemma 2.7 we have $a_{j}^{i}=0$ whenever $i \in\{1, \ldots, n-2\}$ and $j \notin\{i, i+1\}$. Next, by (9) we have that $X_{i}\left(\lambda_{i}\right)=2 a_{i+1}^{i}$ and $X_{i}\left(\lambda_{i}\right)=-2 a_{i-1}^{i-1}$ for any $i \in\{2, \ldots, n-1\}$. So, for any such $i$, by the above equalities and by Lemma 2.10 we obtain that

$$
a_{i+1}^{i}=-a_{i-1}^{i-1}, \quad a_{i}^{i}=a_{i}^{i-1}=0 .
$$

If $n=4$, the various conditions on $a_{i}^{k}$ yield

$$
a_{3}^{2}=-a_{1}^{1}, \quad a_{2}^{1}=a_{2}^{2}=0 .
$$


Therefore the function $\left\{f_{1}, f_{2}, f_{3}, \lambda_{1}, \lambda_{2}, \lambda_{3}, g^{1}, g^{2}, a_{1}^{1}\right\}$ may be linearly indipendent and the dimension of the Lie algebra $\mathcal{M}$ is nine.

If $n>4$ we have that $a_{i}^{k}=0$ for all index $i$ and for all index $k$, then the function are $f_{1}, \ldots, f_{n-1}, \lambda_{1}, \ldots, \lambda_{n-1}, g^{1}, \ldots, g^{n-2}$ are linearly indipendent and $\operatorname{dim} \mathcal{M}=3 n-4$, whence the result.

Acknowledgments. I would like to thank Prof. Filippo De Mari for suggesting the topic and for many useful remarks.

\section{REFERENCES}

[1] T. P. Branson - A. CaP - M. Eastwood - A.R. Gover, Prolongations of geometric overdetermined systems, Internat. J. Math. 17, no. 6 (2006), pp. 641-664.

[2] R. L. BRyant - S.S. Chern - R.B. Gardner - H. Goldschmidt - P.A.GriFFITHS, Exterior differential systems, M.S.R.I. Publications (Springer, 1991).

[3] M. Cowling - A.H. Dooley - A. Korányi - F. Ricci, H-type groups and Iwasawa decompositions, Adv. Math., 87 (1991), pp. 1-41.

[4] M. Cowling - F. De Mari - A. Korányi - H. M. Reimann, Contact and conformal mappings in parabolic geometry. I, Geometriae Dedicata, 111 (2005), pp. 65-86.

[5] M. Cowling - F. De Mari - A. Korányi - H. M. Reimann, Contact and conformal maps on Iwasawa $N$ groups. Rend. Mat. Acc. Lincei s. 9, 13 (2002), 219-232.

[6] A. KAPLAN, Fundamental solutions for a class of hypoelliptic PDE generatted by composition of quadratic forms, Trans. Amer. Math. Soc., 258 (1980),pp. $147-153$.

[7] H. GoLdschmidT, Integrability criteria for systems of nonlinear partial differential equations, J. Differential Geometry, 1 (1967), pp. 269-307.

[8] H. GoLDschmidT, Prolongations of linear partial differential equations. I. A conjecture of Elie Cartan, Ann. Sci. École Norm. Sup. (4), 1 (1968), pp. 417444.

[9] H. GoLDSChmidT, Prolongations of linear partial differential equations. II. Inhomogeneous equations., Ann. Sci. École Norm. Sup. (4), 1 (1968), pp. 617625.

[10] A. KonÁnYI, Multicontact maps: results and conjectures. Lecture notes of Seminario Interdisciplinare di Matematica., IV, pp. 57-63, Lect. Notes Semin. Interdiscip. Mat., IV, S.I.M. Dep. Mat. Univ. Basilicata (Potenza, 2005).

[11] A. KoRÁNYI - H. M. Reimann, Quasiconformal mappings on the Heisenberg group. Invent. Math., 80, n. 2 (1985), pp. 309-338.

[12] F. De Mari - M. A. Shayman, Generalized Eulerian numbers and the topology of the Hessenberg variety of a matrix, Acta Appl. Math., 12 (1988), pp. 213-235.

[13] F. De Mari - C. Procesi - M. A. Shayman, Hessenberg varieties, Trans. Amer. Math. Soc., 332 (1992), pp. 529-534. 
[14] M. EASTWOOD, Real 3-dimensional CR geometry, unpublished manuscript (2004).

[15] T. MoRimoto, Geometric structures on filtered manifolds, Hokkaido Math.J., 22, no. 3 (1994), pp. 263-347.

[16] A. OtTAZZI, Multicontact Vector Fields on Hessenberg Manifolds, Journal of Lie Theory, 15 (2005), pp. 357-377.

[17] P. Pansu, Métriques de Carnot-Carathéodory et quasiisométries des espaces symétriques de rang un, Ann. of Math. (2), 129, no. 1 (1989), pp. 1-60.

[18] H. M. Reimann, Rigidity of H-type groups, Math. Z., 237 (2001), pp. 697-725.

[19] N. TANAKA, On differential systems, graded Lie algebras and pseudo-groups, J. Math. Kyoto Univ., 10 (1970), pp. 1-82.

[20] K. Yamaguchi, Differential systems associated with simple graded Lie algebras. In: Progress in differential geometry, Adv. Stud. Pure Math., 22, Math. Soc. Japan (Tokyo, 1993), pp. 413-494.

[21] T. Yatsui, On pseudo-product graded Lie algebras, Hokkaido Math. J., 17, no. 3 (1988), pp. 333-343.

[22] B. WARHURST, Contact and quasiconformal mappings on real model filiform groups, Bull. Austral. Math. Soc., 68, no. 2 (2003), pp. 329-343.

[23] B. Warhurst, Jet spaces as nonrigid Carnot groups, J. Lie Theory, 15, no. 1 (2005), pp. 341-356.

[24] B. WarhuRst, Tanaka prolongation of free Lie algebras, Geom. Dedicata, 130 (2007), pp. 59-69.

Manoscritto pervenuto in redazione il 9 agosto 2008. 
